\title{
2 Aqueous Elemental Mercury Production versus Mercury Inventories in the Lake Michigan Airshed: Sorting Out the Spatial and Diel Controls of Mercury Gradients in Air and Water
}

Ryan F. Lepak*a,b,c, Michael T. Tate ${ }^{b}$, Benjamin D. Peterson ${ }^{a}$, Jacob M. Ogorek ${ }^{b}$, John F. DeWild ${ }^{b}$,

6 James P. Hurley, and David P. Krabbenhoft ${ }^{b}$

7

aEnvironmental Chemistry and Technology Program, University of Wisconsin-Madison, $660 \mathrm{~N}$.

9 Park Street, Madison, WI 53706, USA

10 bU.S. Geological Survey, Upper Midwest Water Science Center, USGS Mercury Research

11 Laboratory, 8505 Research Way, Middleton, Wisconsin 53562, USA

12 CU.S. EPA Office of Research and Development, Center for Computational Toxicology and

13 Exposure, Great Lakes Toxicology and Ecology Division, 6201 Congdon Blvd, Duluth, MN 55804, 14 USA

dUniversity of Wisconsin Aquatic Sciences Center, 1975 Willow Dr., Madison, WI 53706, USA

Disclaimer: Any use of trade, firm, or product names is for descriptive purposes only and does not imply endorsement by the U.S. Government.

*corresponding author

27 *Ryan Lepak, U.S. EPA Office of Research and Development, Center for Computational

28 Toxicology and Exposure, Great Lakes Toxicology and Ecology Division, 6201 Congdon Blvd,

29 Duluth, MN 55804, USA - rlepak@wisc.ed 
30 Supporting Information:

31 S2-Text.

32 S2 - Figure S1 - Compared DGM flow through and manual methods

33 S3 to S15 - Figure S2 to S26 - Vertical water chemistry profiles and Hg measurements

34 S16 - Figure S27 - Satellite imagery of Lake Michigan during the cruise

35 S17 to S21 - Table S1 - Continuous surface measurements of Lake Michigan

36 S22 - Table S2 - Paired atmospheric Hg and DGM measurements

37 S23 - Table S3 - Vertical mercury and DOC measurements

38 S24 - Citations

39

40

41

42

43

44

45

46

47

48

49

50

51

52

53

54

55

56

57

58 
For the calculation of dissolved gaseous mercury (DGM) mass in the photic and aphotic zones of the main basin and Green Bay, the Surface Volume tool in ESRI TM's 3-D analyst extension was used to

62 calculate volumes from a digital elevation bathymetric map for Lake Michigan (National Geophysical

63 Data Center). ${ }^{1}$ UV and photosynthetic active radiation (PAR) extinction depths (7 mand $26.3 \mathrm{~m}$,

64 respectively) were used at the lower boundary of the photic zones.

65

Figure S1: Comparison of the custom-designed flow through system to manual purge and trap dissolved

67 gaseous elemental $\mathrm{Hg}$ (DGM) concentrations as well as the percent differences. Manual DGM samples were collected at the vertical profile stations and the flow through system ran continuously while on station thus producing many measurements in a single location. Error bars represent the 1 standard deviation of the continuous measurements.

71

Percent difference DGM methods

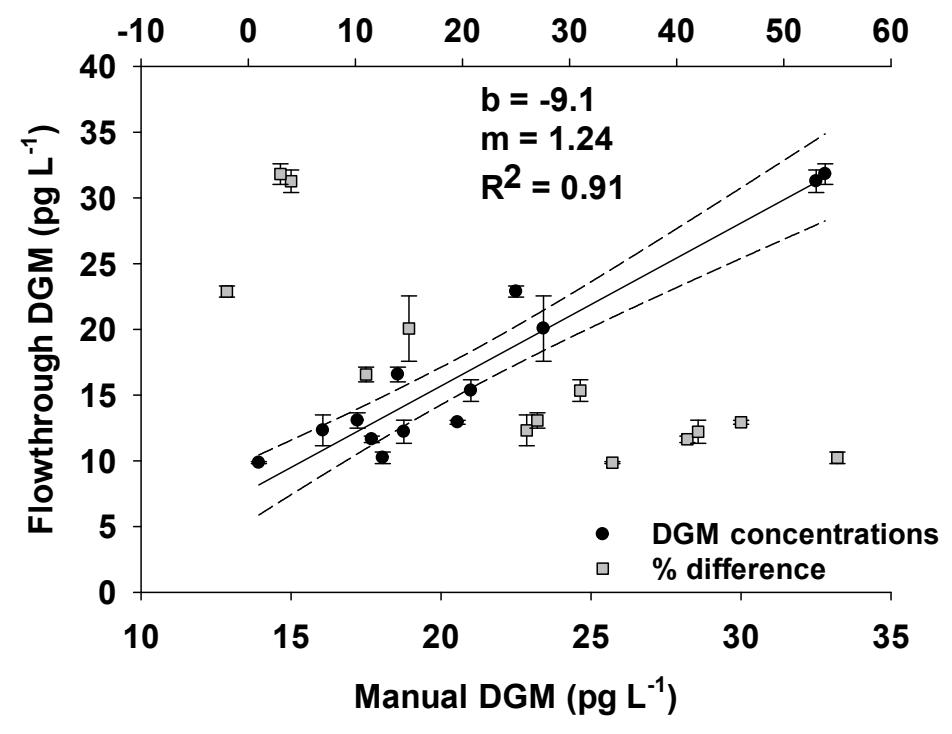


Figure S2: Site - MI100, DGM saturation profile, water quality measurements, and biological fluorescence profiles from the U.S. Environmental Protection Agency's R/V Lake Guardian. PAR is the photosynthetically active radiation. Profile data may be found on the EPA Central Data Exchange.
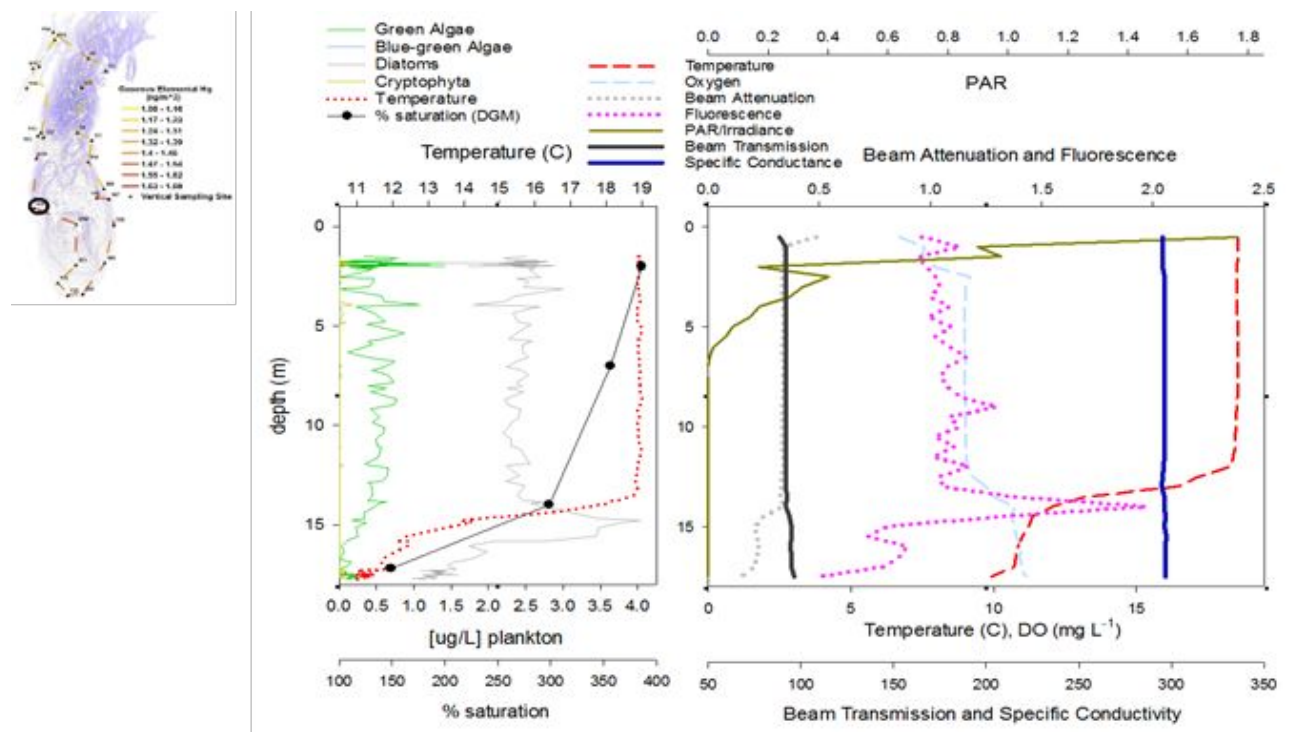

Figure S3: Site - MI18M, DGM saturation profile, water quality measurements, and biological fluorescence profiles from the U.S. Environmental Protection Agency's R/V Lake Guardian. PAR is the photosynthetically active radiation. Profile data may be found on the EPA Central Data Exchange.

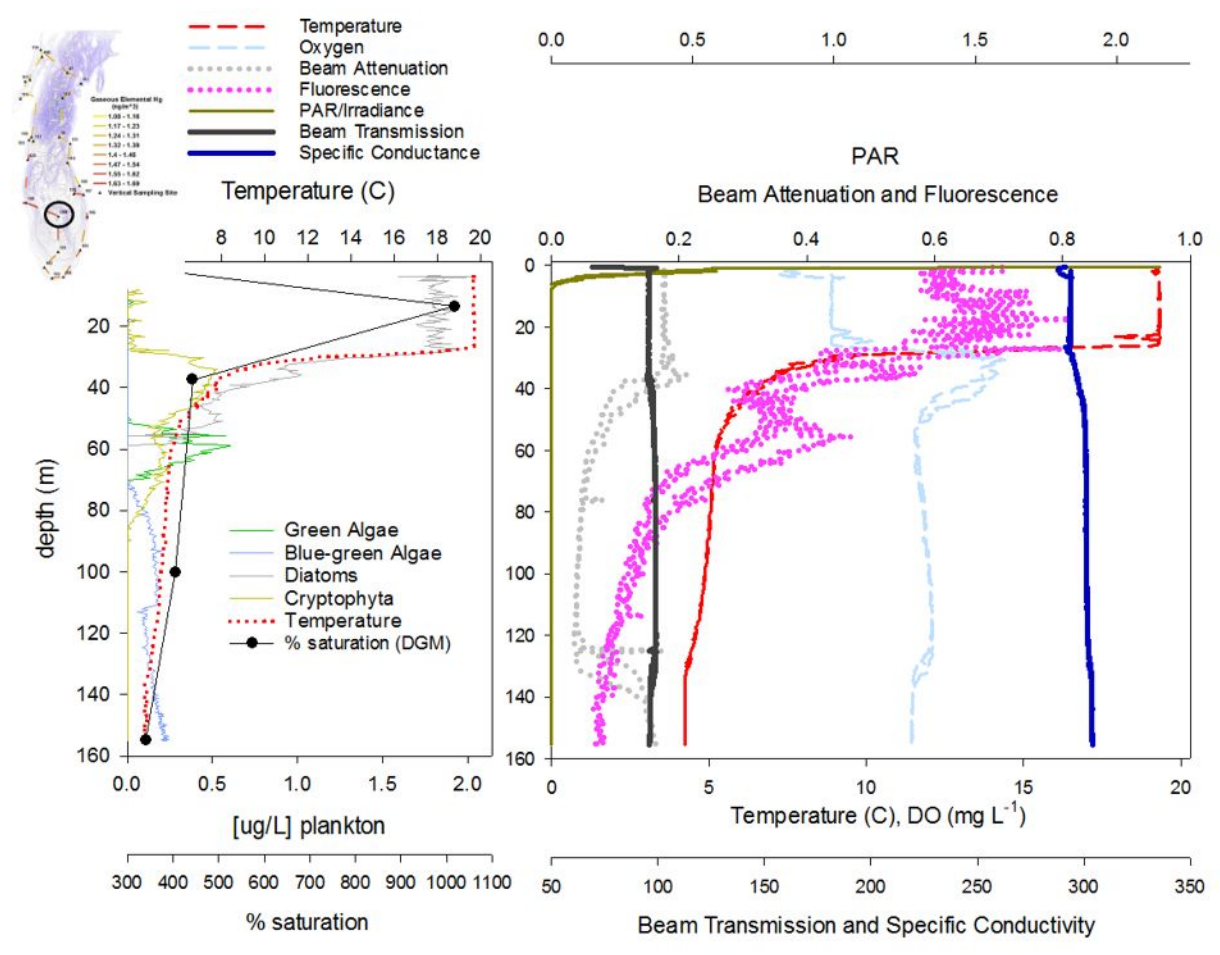


Figure S3: Site - MI101, DGM saturation profile, water quality measurements, and biological fluorescence profiles from the U.S. Environmental Protection Agency's R/V Lake Guardian. PAR is the photosynthetically active radiation. Profile data may be found on the EPA Central Data Exchange.
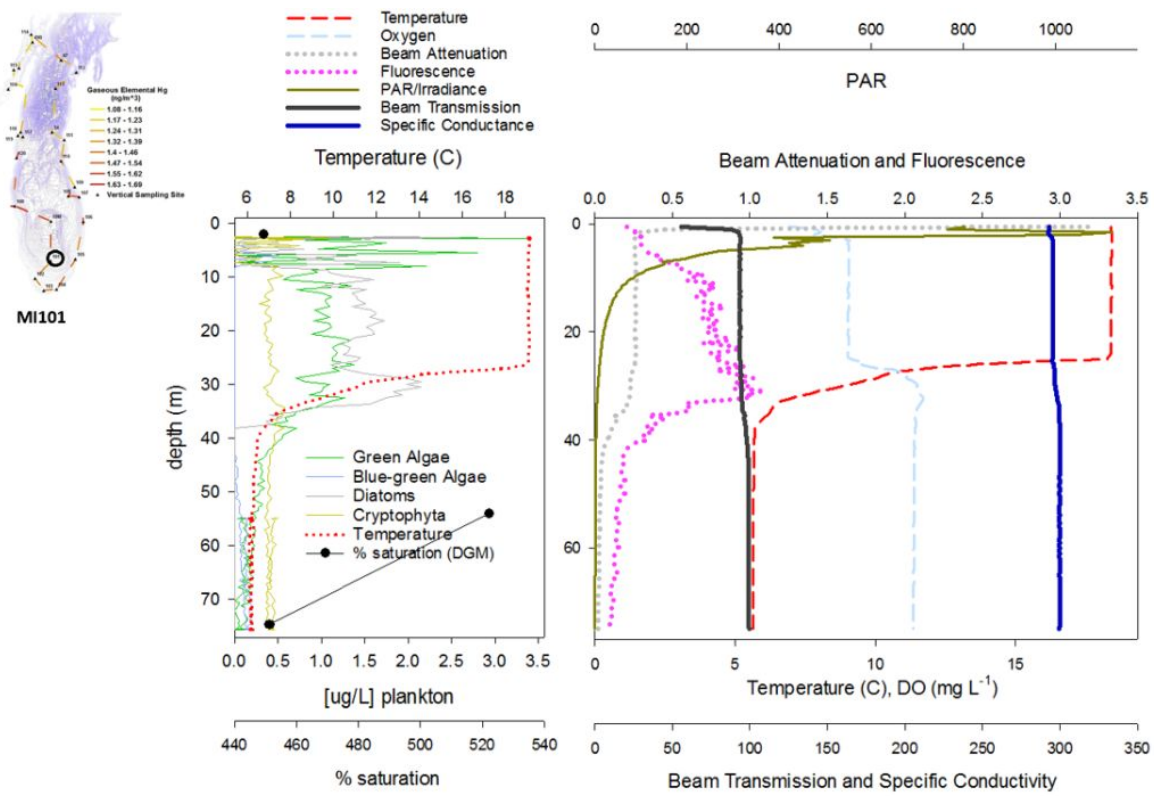

Figure S4: Site - MI102, DGM saturation profile, water quality measurements, and biological fluorescence profiles from the U.S. Environmental Protection Agency's R/V Lake Guardian. PAR is the photosynthetically active radiation. Profile data may be found on the EPA Central Data Exchange.
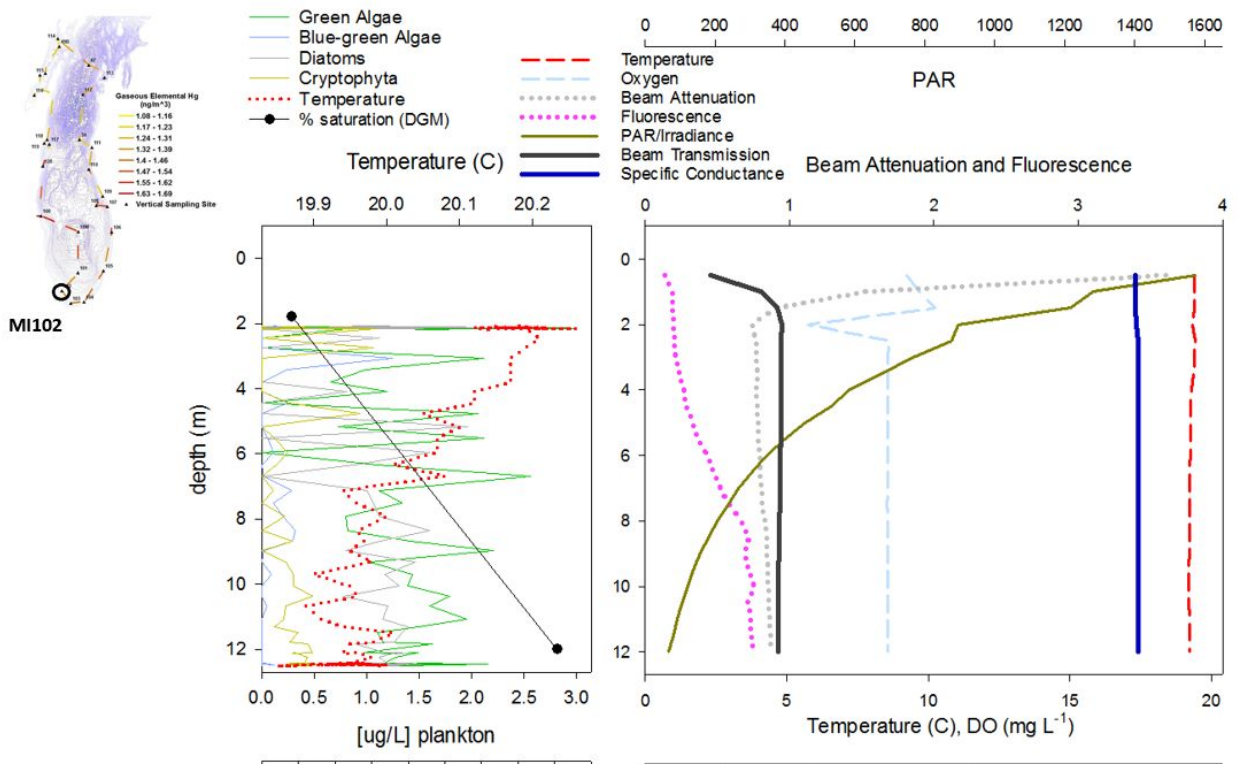

260280300320340360380400420440 $\%$ saturation

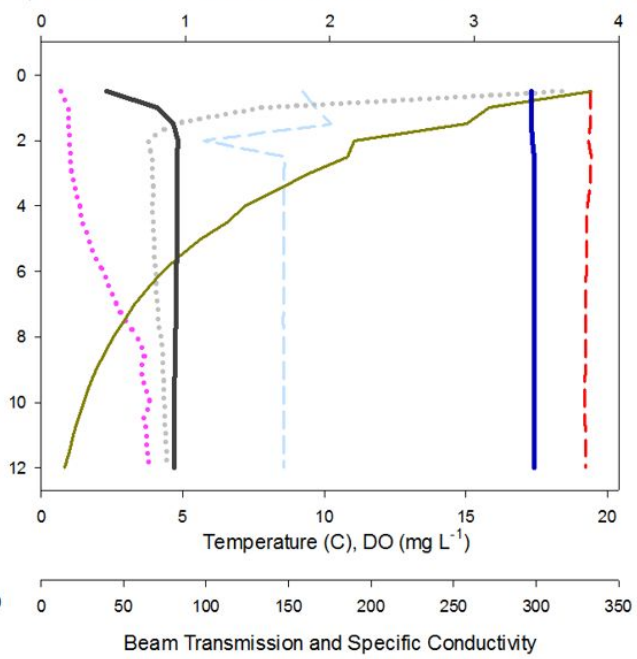


Figure S5: Site - MI103, DGM saturation profile, water quality measurements, and biological fluorescence profiles from the U.S. Environmental Protection Agency's R/V Lake Guardian. PAR is the photosynthetically active radiation. Profile data may be found on the EPA Central Data Exchange.
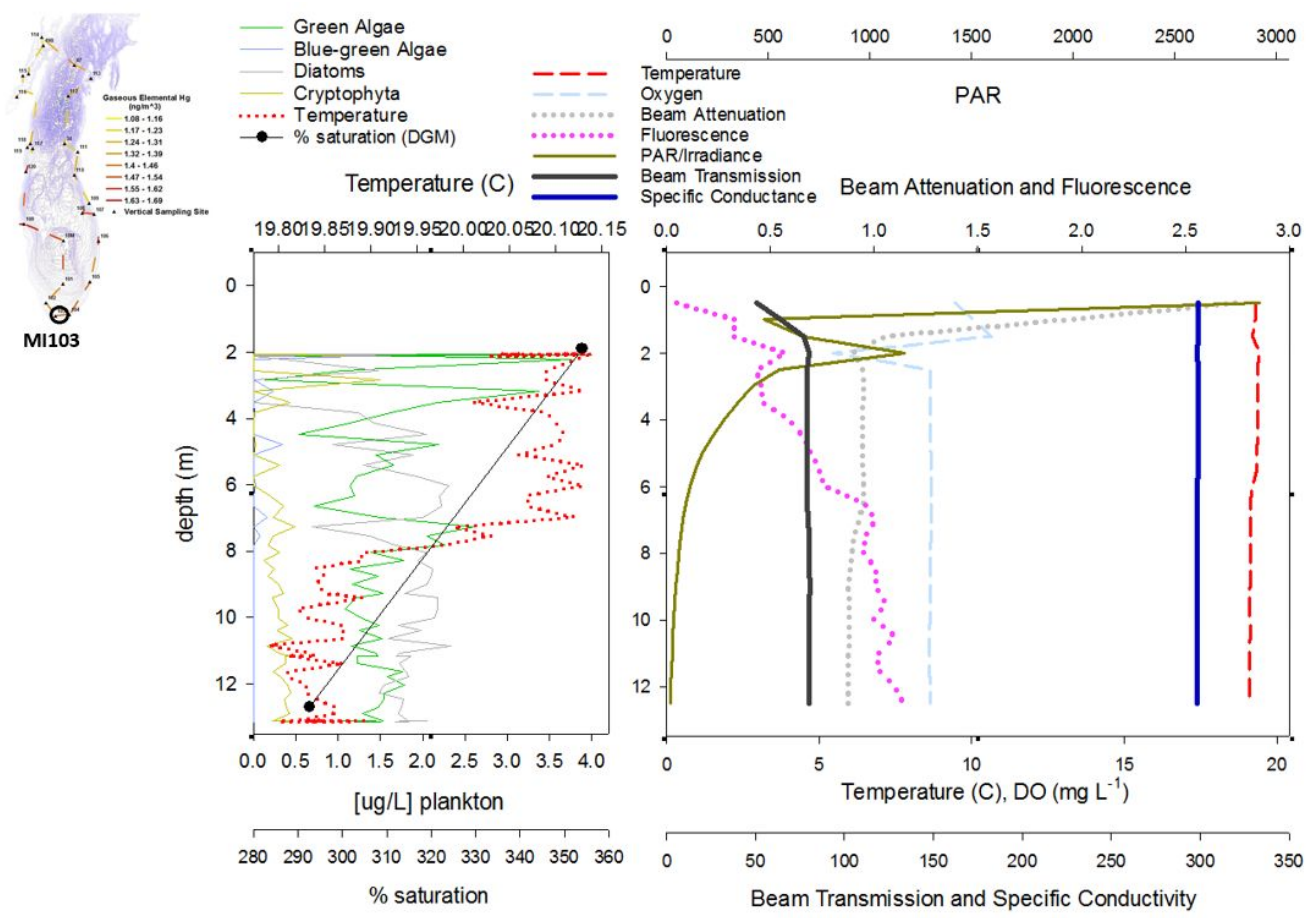

Figure S6: Site - MI104, DGM saturation profile, water quality measurements, and biological fluorescence profiles from the U.S. Environmental Protection Agency's R/V Lake Guardian. PAR is the photosynthetically active radiation. Profile data may be found on the EPA Central Data Exchange.
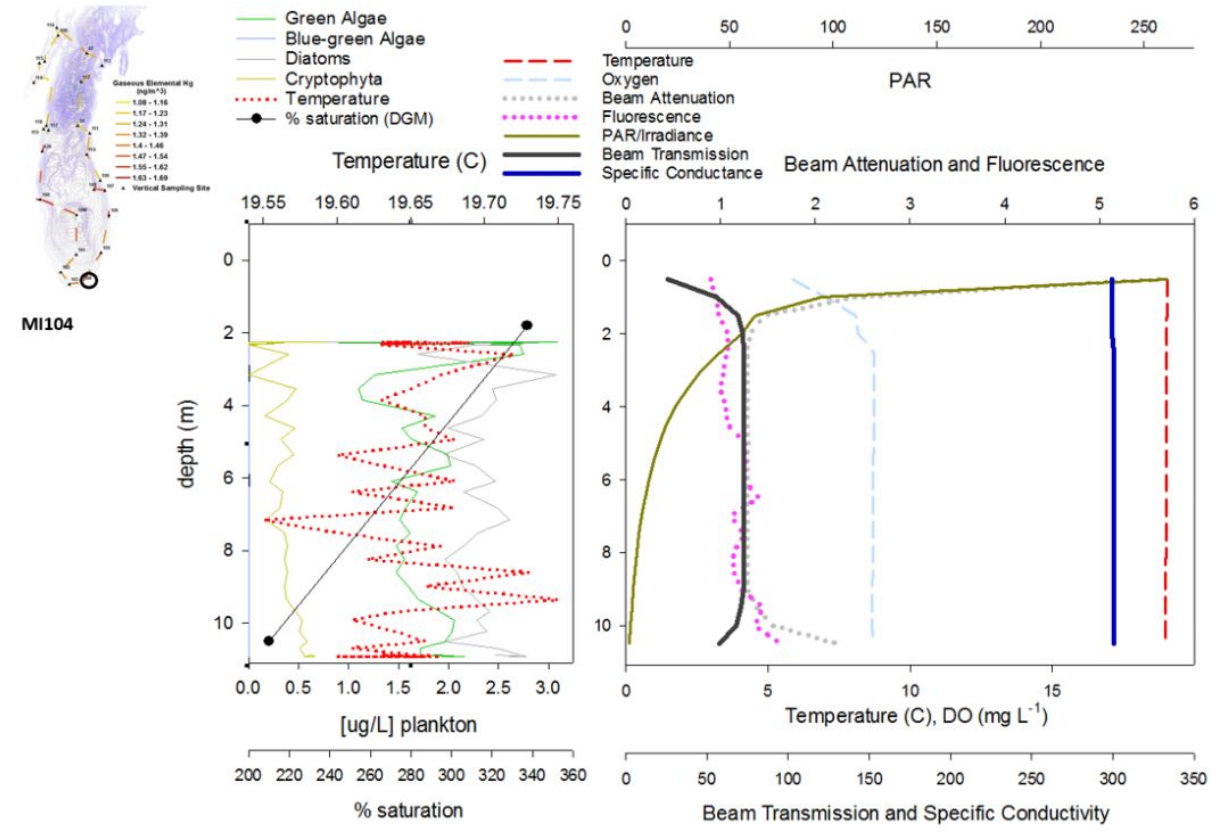
Figure S7: Site - MI105, DGM saturation profile, water quality measurements, and biological fluorescence profiles from the U.S. Environmental Protection Agency's R/V Lake Guardian. PAR is the photosynthetically active radiation. Profile data may be found on the EPA Central Data Exchange.
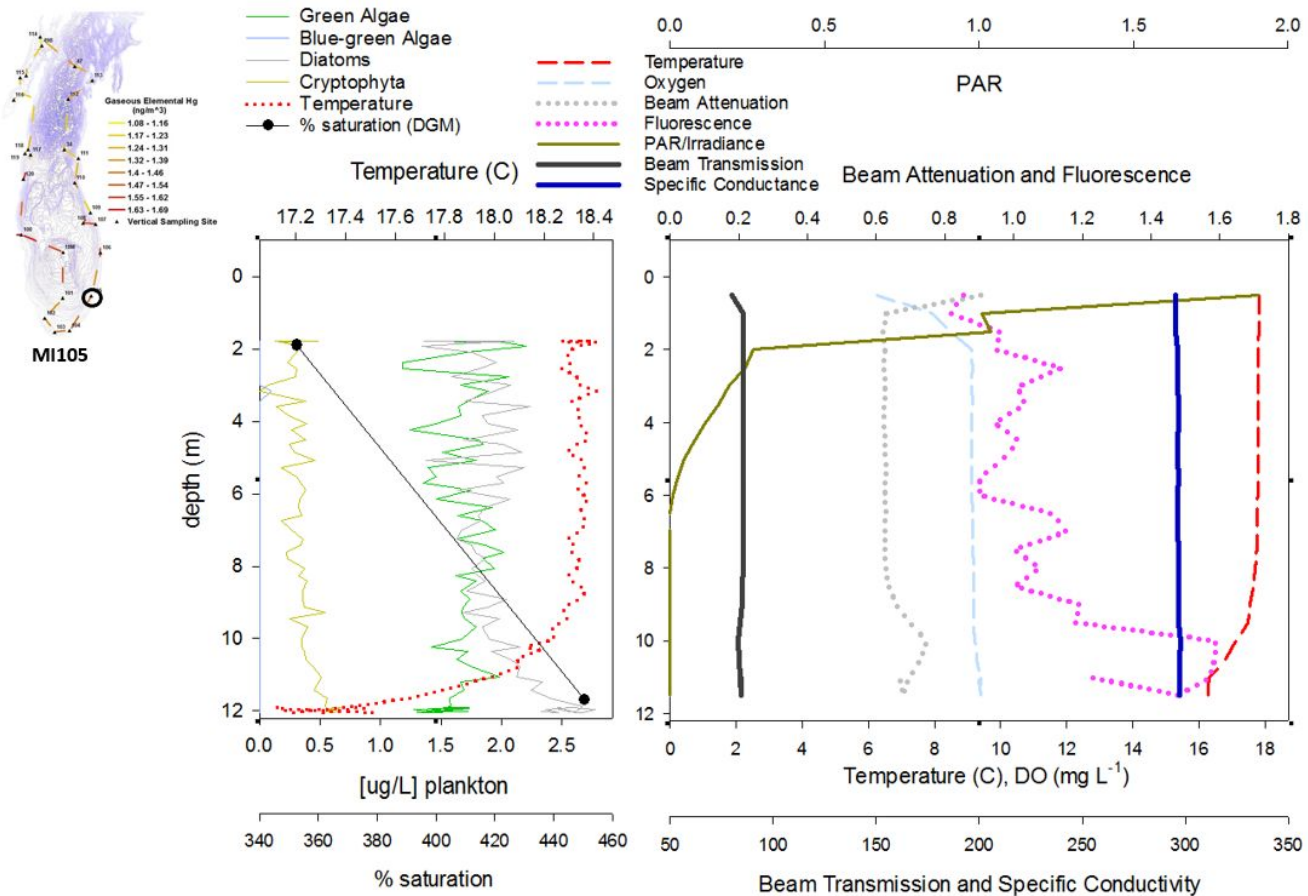

Figure S8: Site - MI106, DGM saturation profile, water quality measurements, and biological fluorescence profiles from the U.S. Environmental Protection Agency's R/V Lake Guardian. PAR is the photosynthetically active radiation. Profile data may be found on the EPA Central Data Exchange.

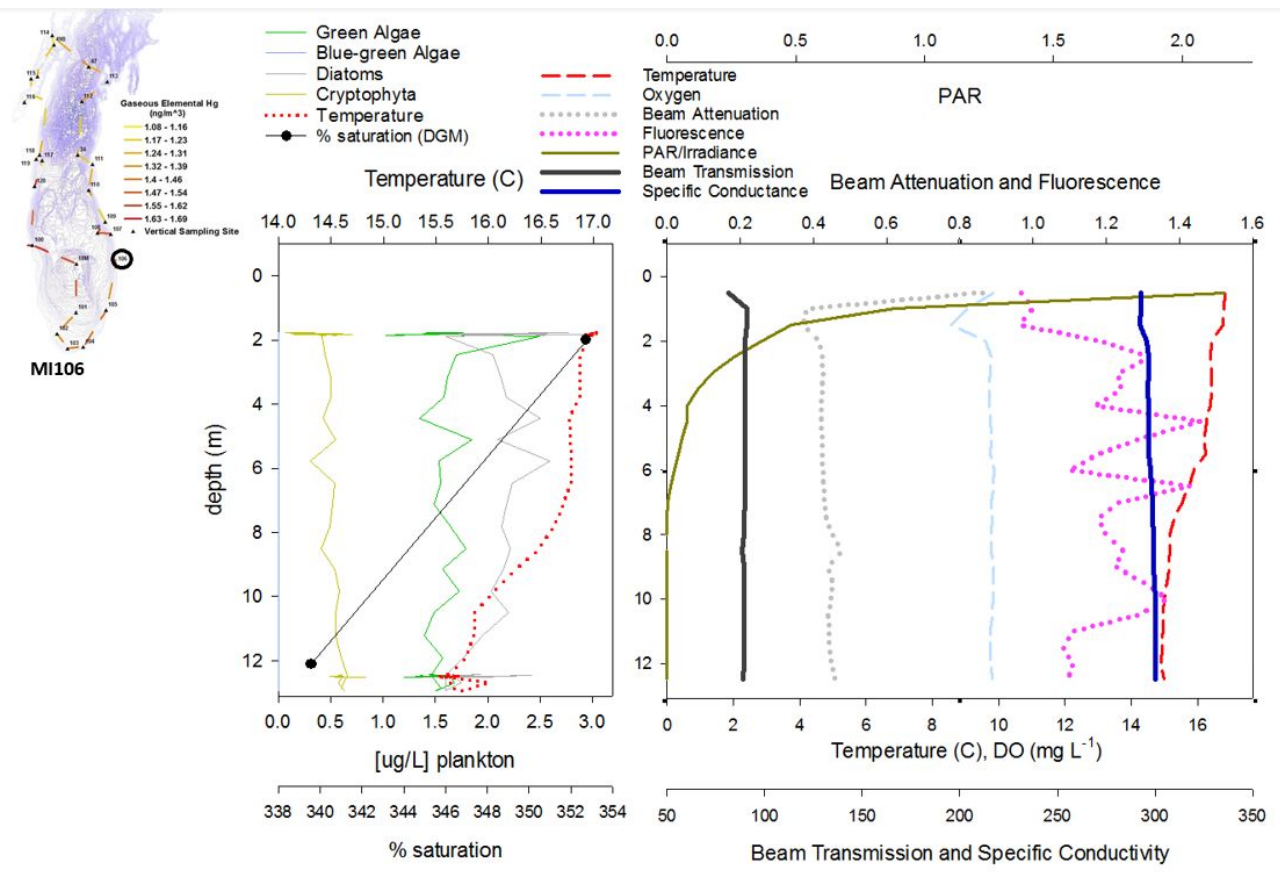


Figure S9: Site - MI107, DGM saturation profile, water quality measurements, and biological fluorescence profiles from the U.S. Environmental Protection Agency's R/V Lake Guardian. PAR is the photosynthetically active radiation. Profile data may be found on the EPA Central Data Exchange.

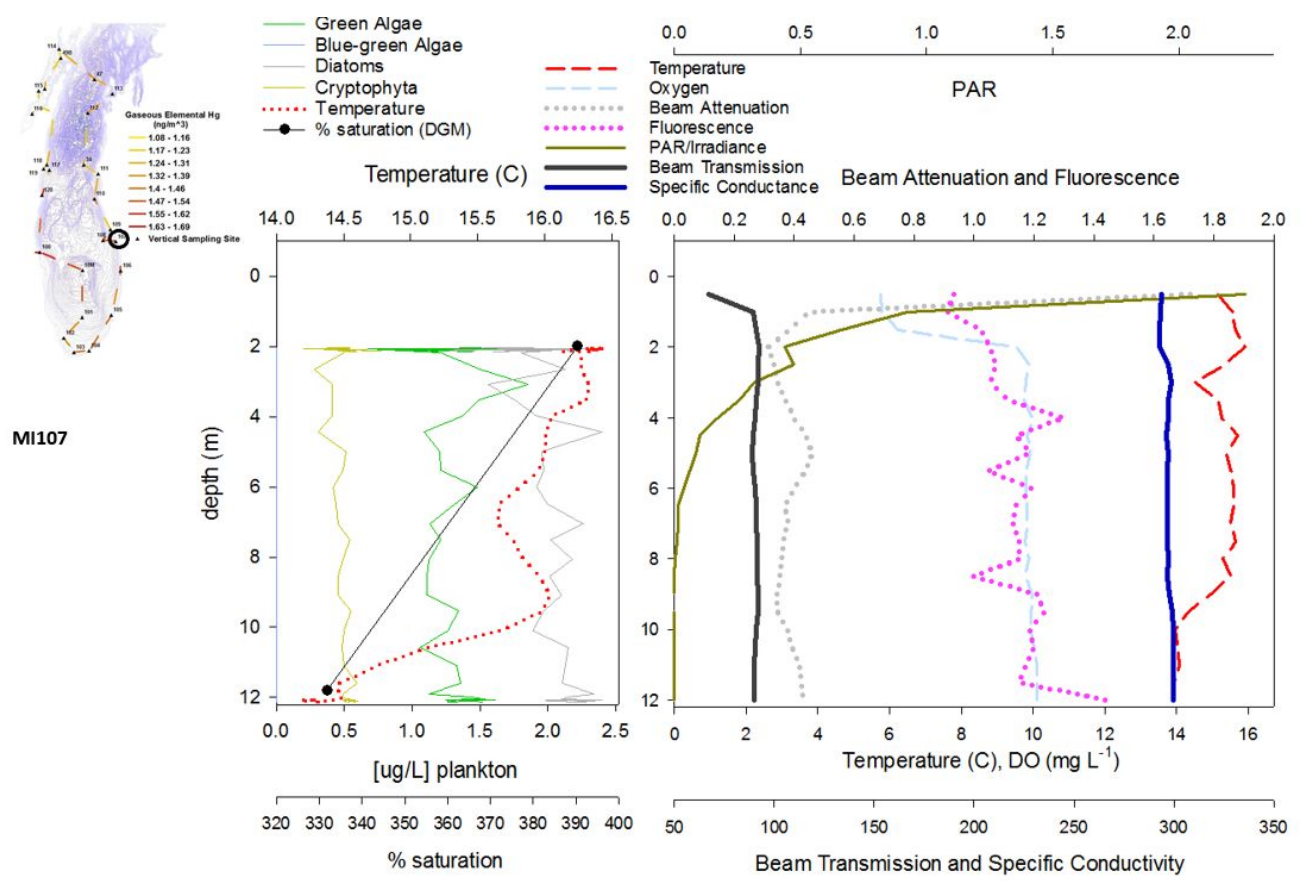

Figure S10: Site - MI108, DGM saturation profile, water quality measurements, and biological fluorescence profiles from the U.S. Environmental Protection Agency's R/V Lake Guardian. PAR is the photosynthetically active radiation. Profile data may be found on the EPA Central Data Exchange.

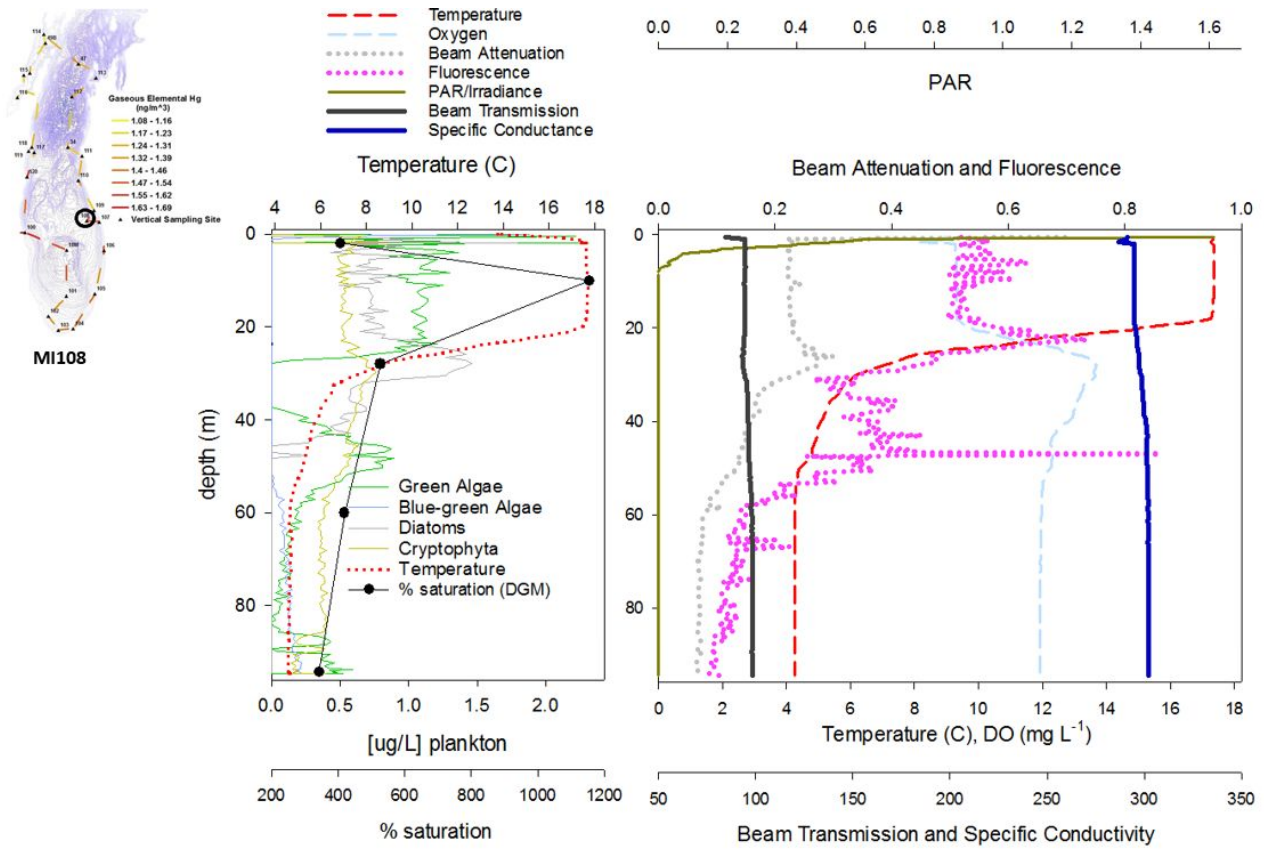


Figure S11: Site - MI109, DGM saturation profile, water quality measurements, and biological fluorescence profiles from the U.S. Environmental Protection Agency's R/V Lake Guardian. PAR is the photosynthetically active radiation. Profile data may be found on the EPA Central Data Exchange.

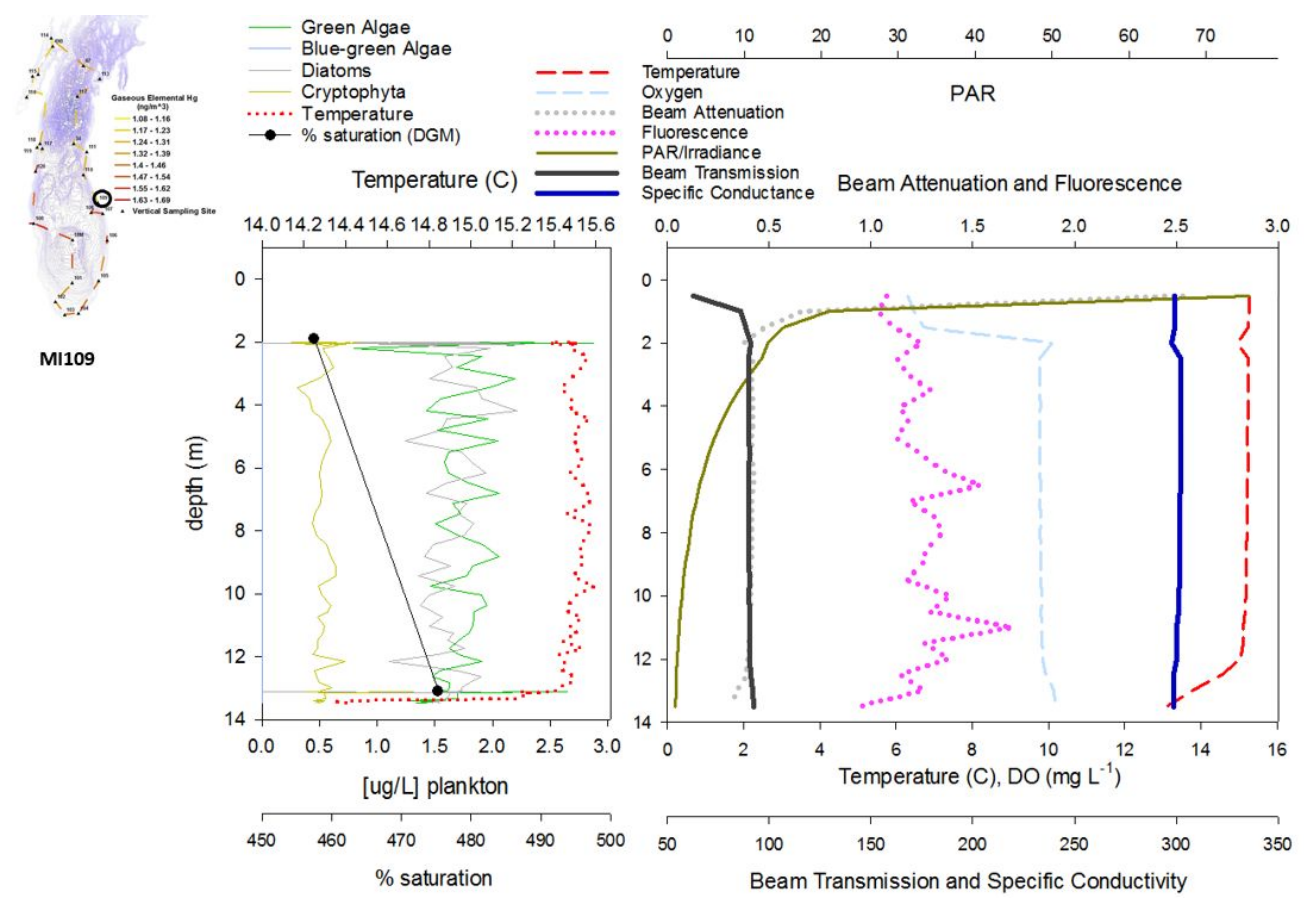

Figure S12: Site - MI110, DGM saturation profile, water quality measurements, and biological fluorescence profiles from the U.S. Environmental Protection Agency's R/V Lake Guardian. PAR is the photosynthetically active radiation. Profile data may be found on the EPA Central Data Exchange.

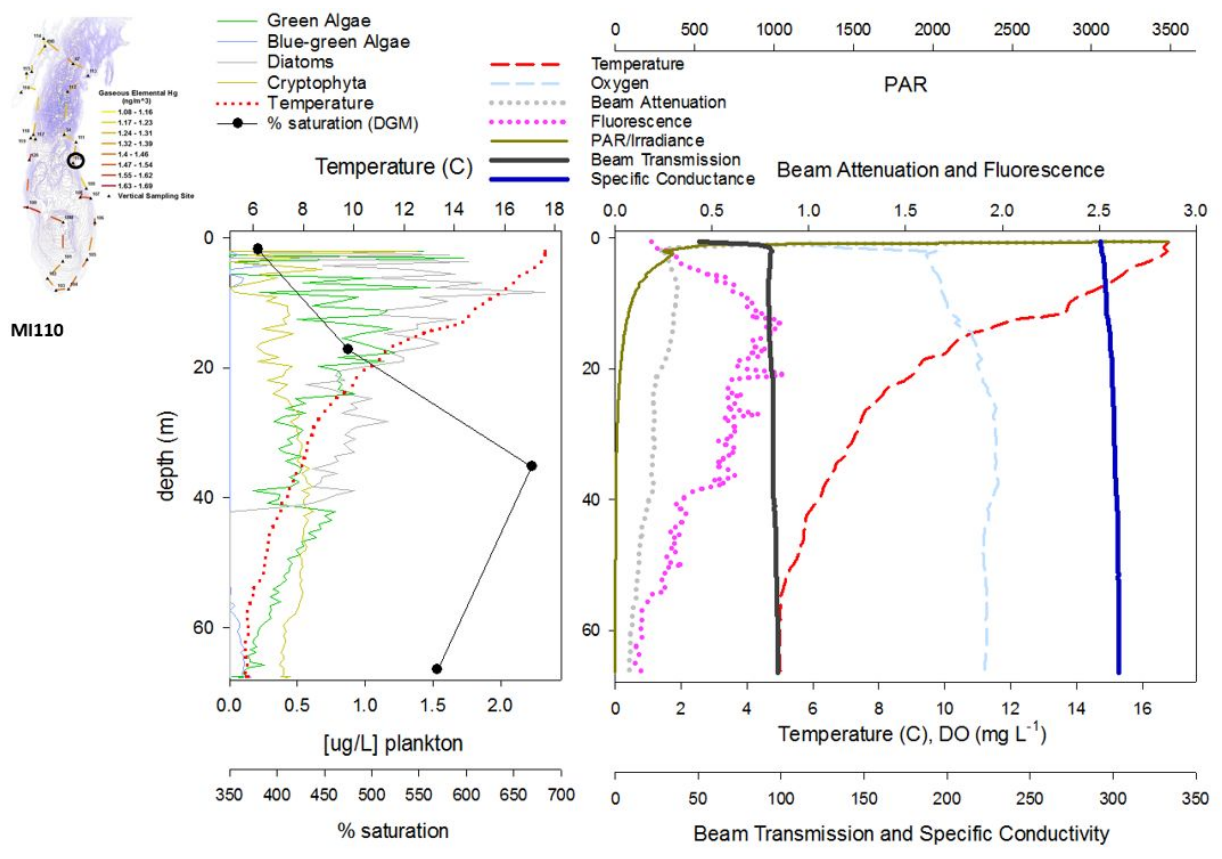


Figure S13: Site - MI111, DGM saturation profile, water quality measurements, and biological fluorescence profiles from the U.S. Environmental Protection Agency's R/V Lake Guardian. PAR is the photosynthetically active radiation. Profile data may be found on the EPA Central Data Exchange.

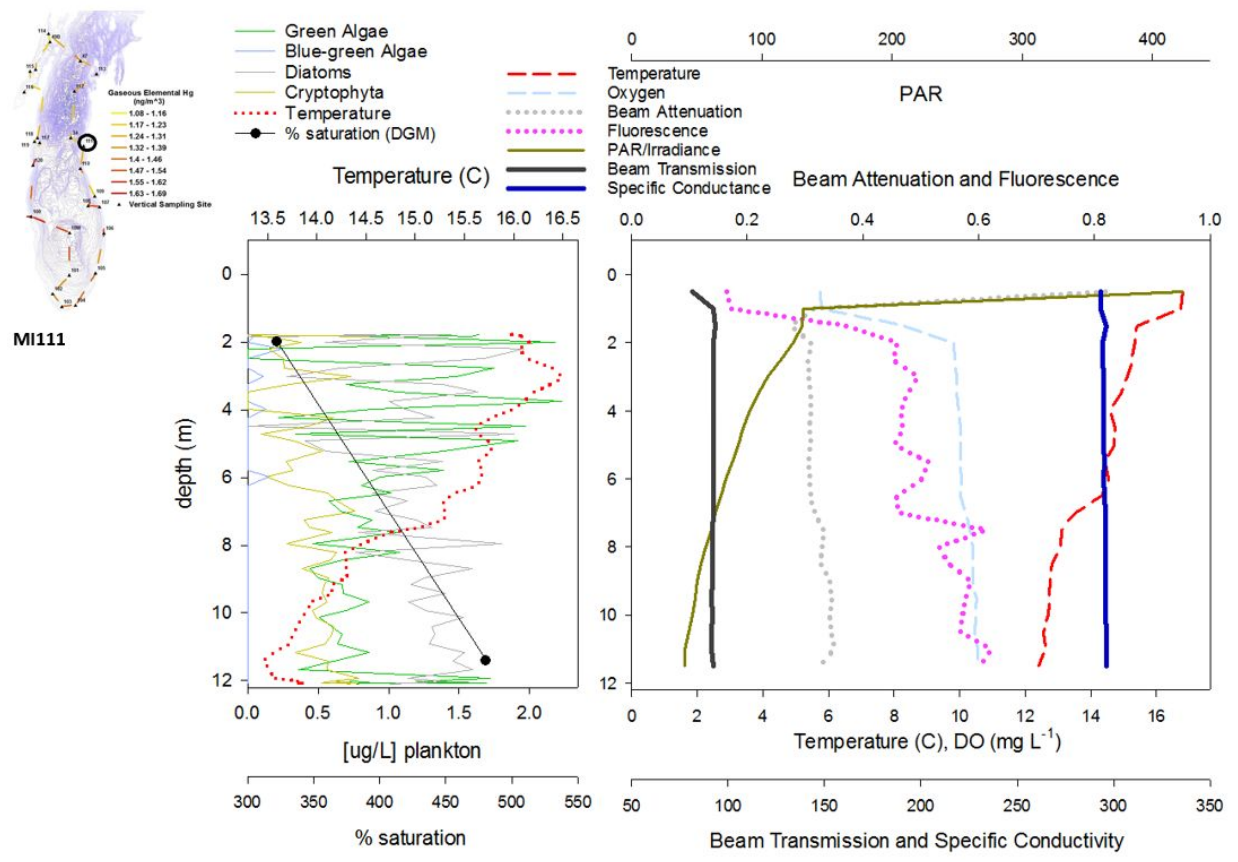

Figure S14: Site - MI34, DGM saturation profile, water quality measurements, and biological fluorescence profiles from the U.S. Environmental Protection Agency's R/V Lake Guardian. PAR is the photosynthetically active radiation. Profile data may be found on the EPA Central Data Exchange.

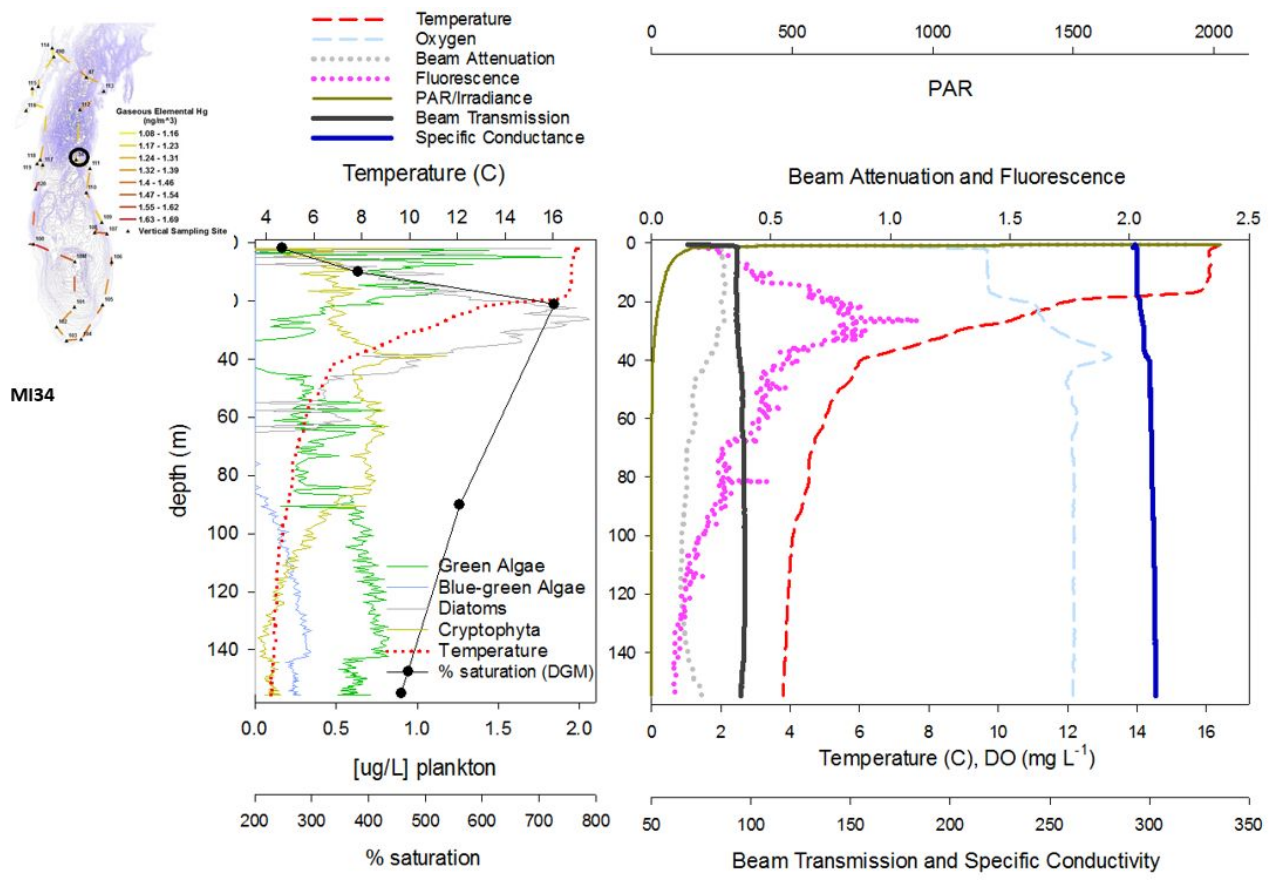


Figure S15: Site - MI112, DGM saturation profile, water quality measurements, and biological fluorescence profiles from the U.S. Environmental Protection Agency's R/V Lake Guardian. PAR is the photosynthetically active radiation. Profile data may be found on the EPA Central Data Exchange.

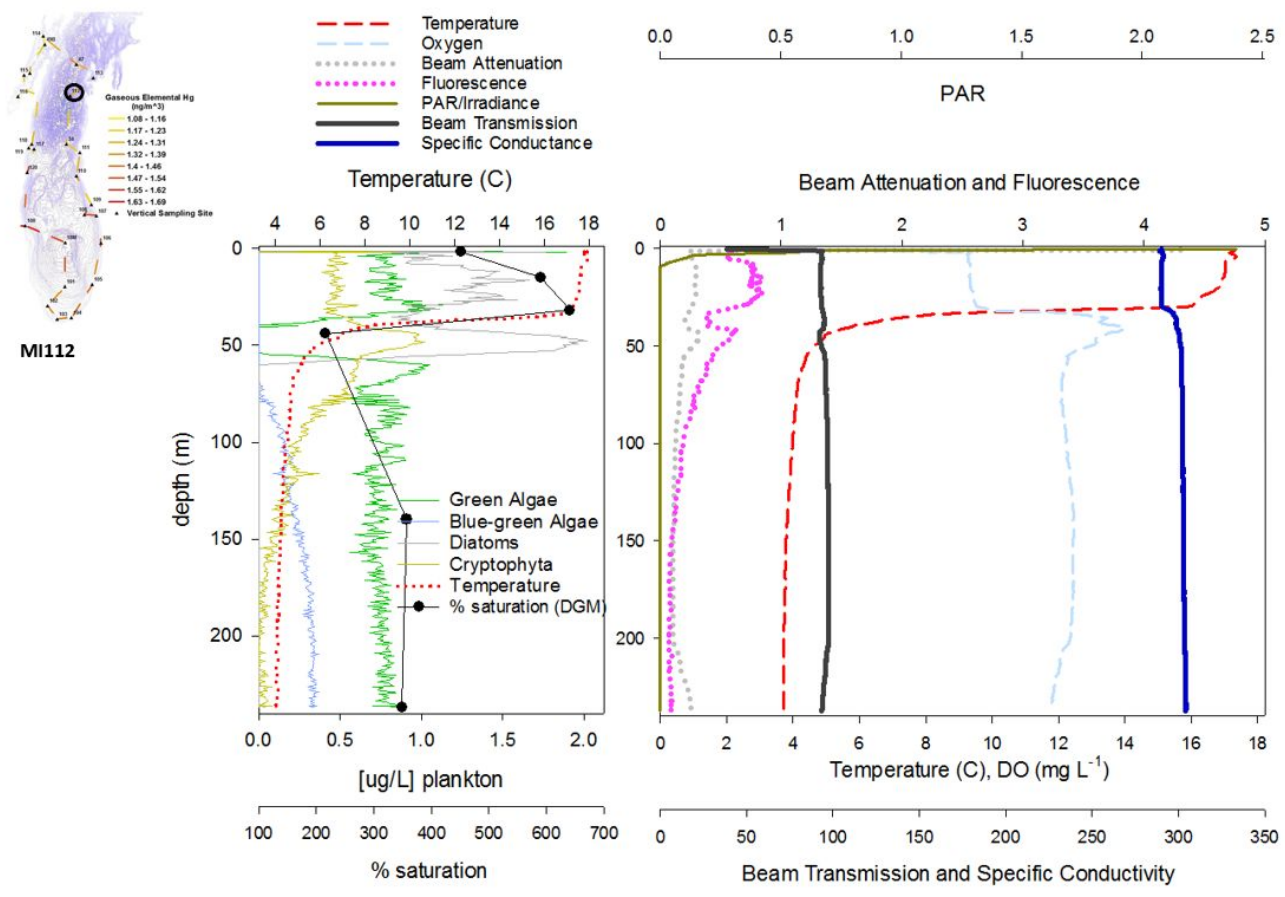

Figure S16: Site - MI113, DGM saturation profile, water quality measurements, and biological fluorescence profiles from the U.S. Environmental Protection Agency's R/V Lake Guardian. PAR is the photosynthetically active radiation. Profile data may be found on the EPA Central Data Exchange.

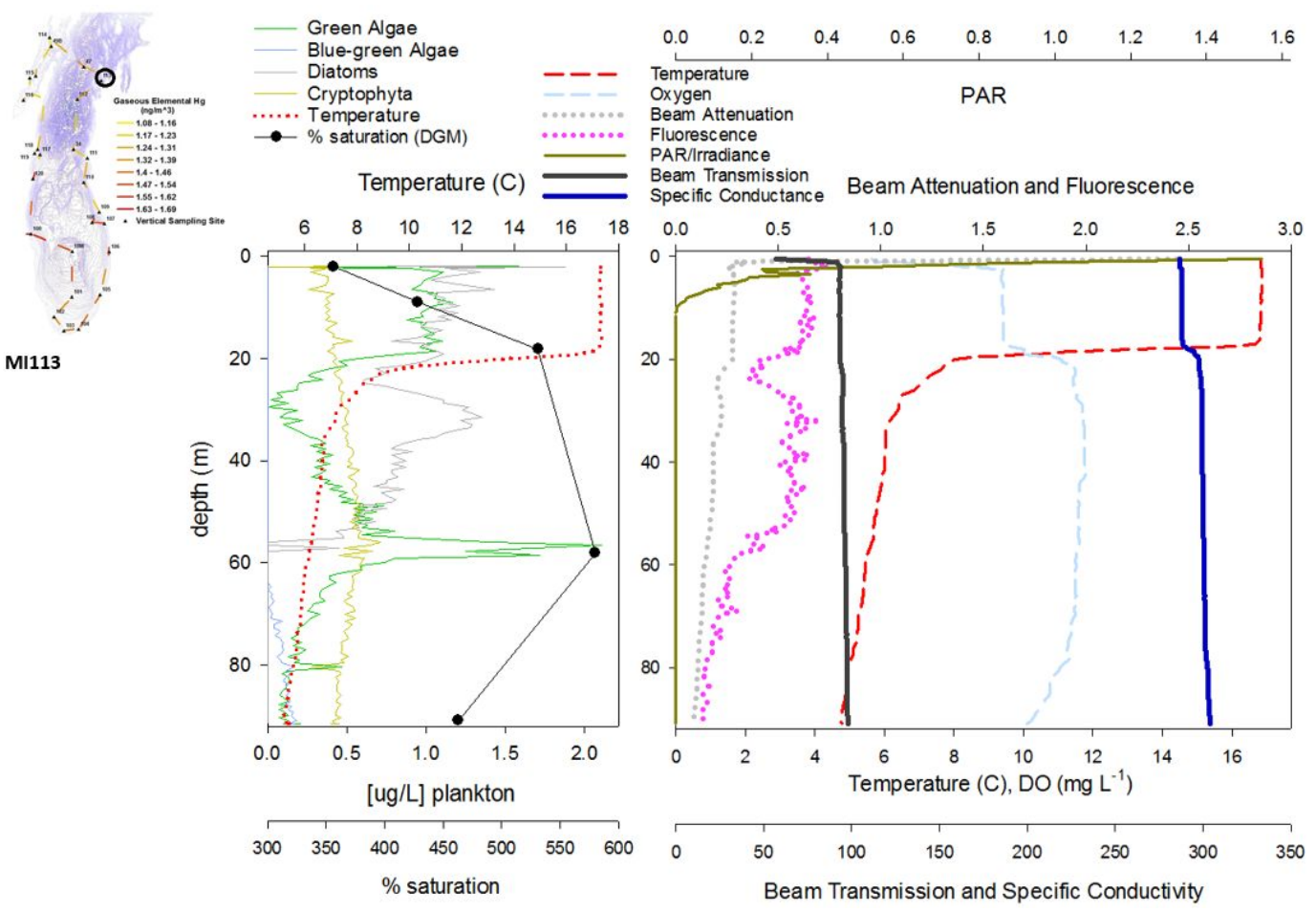


Figure S17: Site - MI47, DGM saturation profile, water quality measurements, and biological fluorescence profiles from the U.S. Environmental Protection Agency's R/V Lake Guardian. PAR is the photosynthetically active radiation. Profile data may be found on the EPA Central Data Exchange.

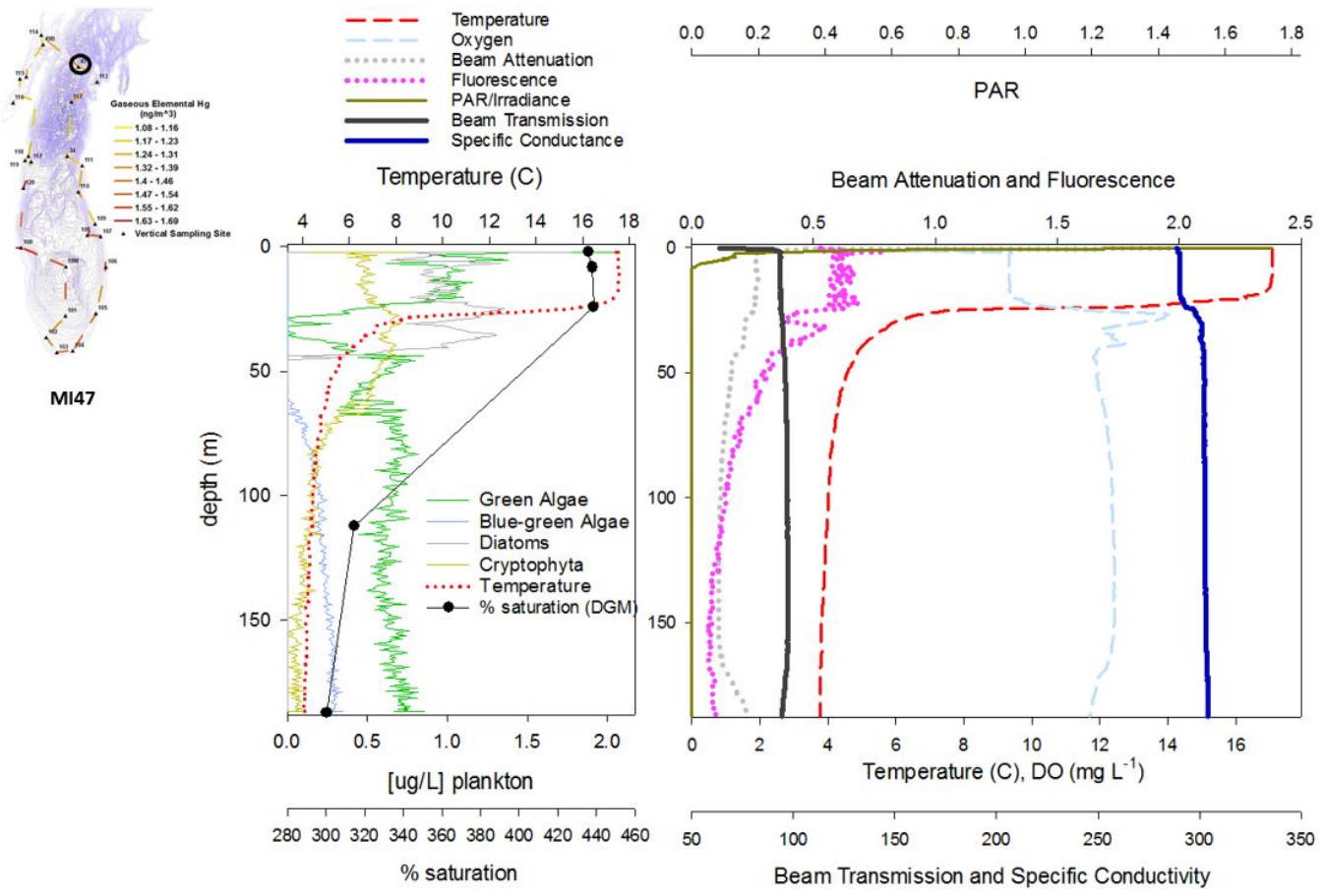

Figure S18: Site - MI114, DGM saturation profile, water quality measurements, and biological fluorescence profiles from the U.S. Environmental Protection Agency's R/V Lake Guardian. PAR is the photosynthetically active radiation. Profile data may be found on the EPA Central Data Exchange.

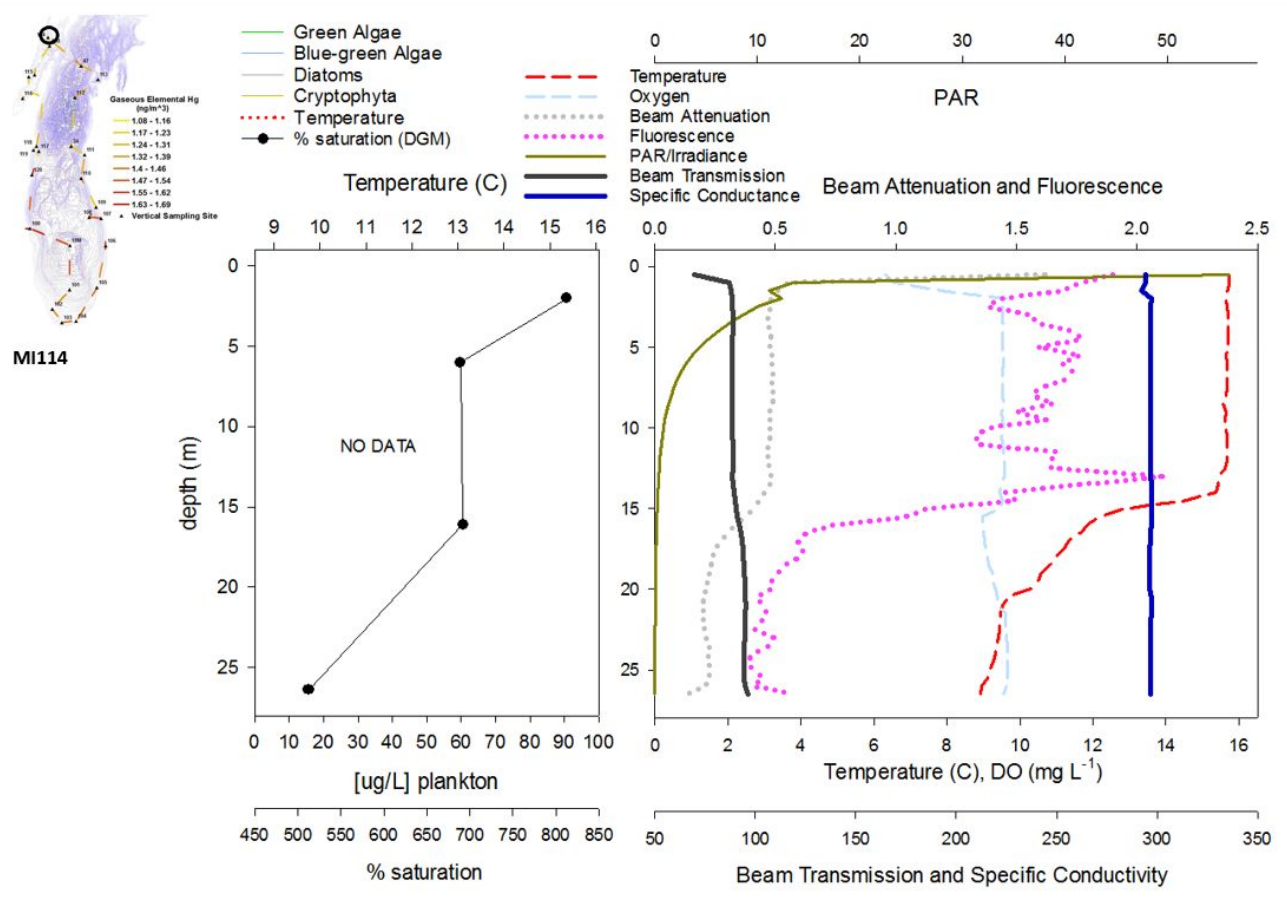


Figure S19: Site - MI49B, DGM saturation profile, water quality measurements, and biological fluorescence profiles from the U.S. Environmental Protection Agency's R/V Lake Guardian. PAR is the photosynthetically active radiation. Profile data may be found on the EPA Central Data Exchange.
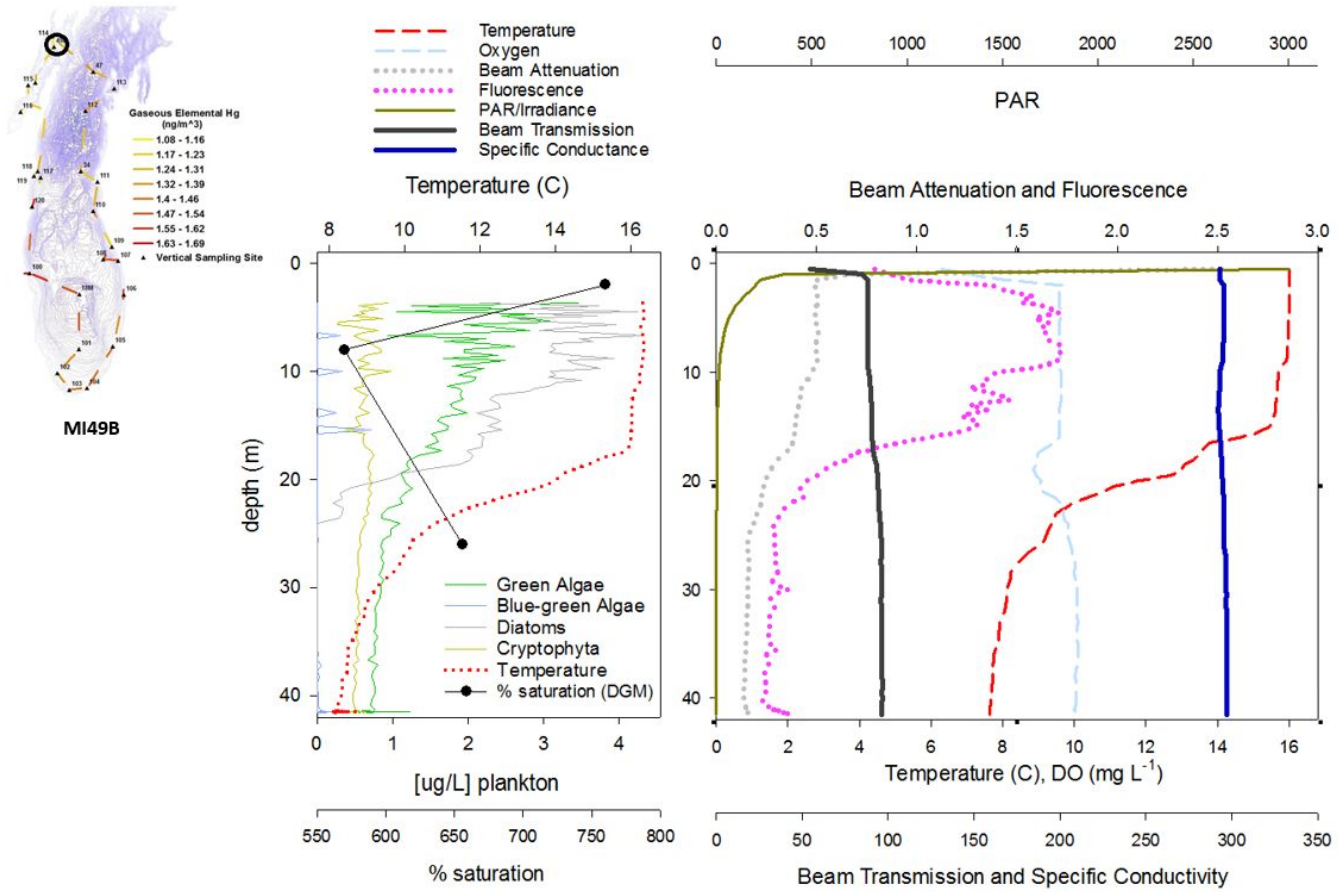

Figure S20: Site - MI50B, DGM saturation profile, water quality measurements, and biological fluorescence profiles from the U.S. Environmental Protection Agency's R/V Lake Guardian. PAR is the photosynthetically active radiation. Profile data may be found on the EPA Central Data Exchange.

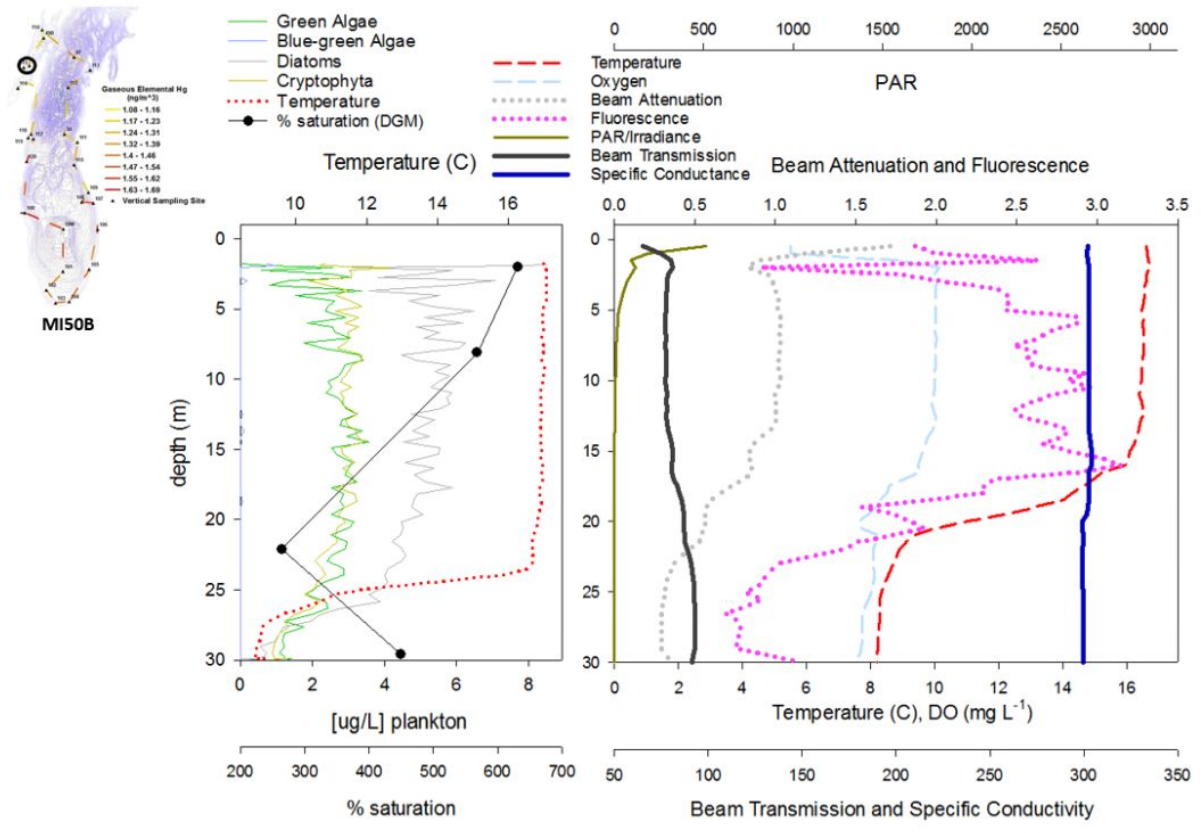


Figure S21: Site - MI115, DGM saturation profile, water quality measurements, and biological fluorescence profiles from the U.S. Environmental Protection Agency's R/V Lake Guardian. PAR is the photosynthetically active radiation. Profile data may be found on the EPA Central Data Exchange.
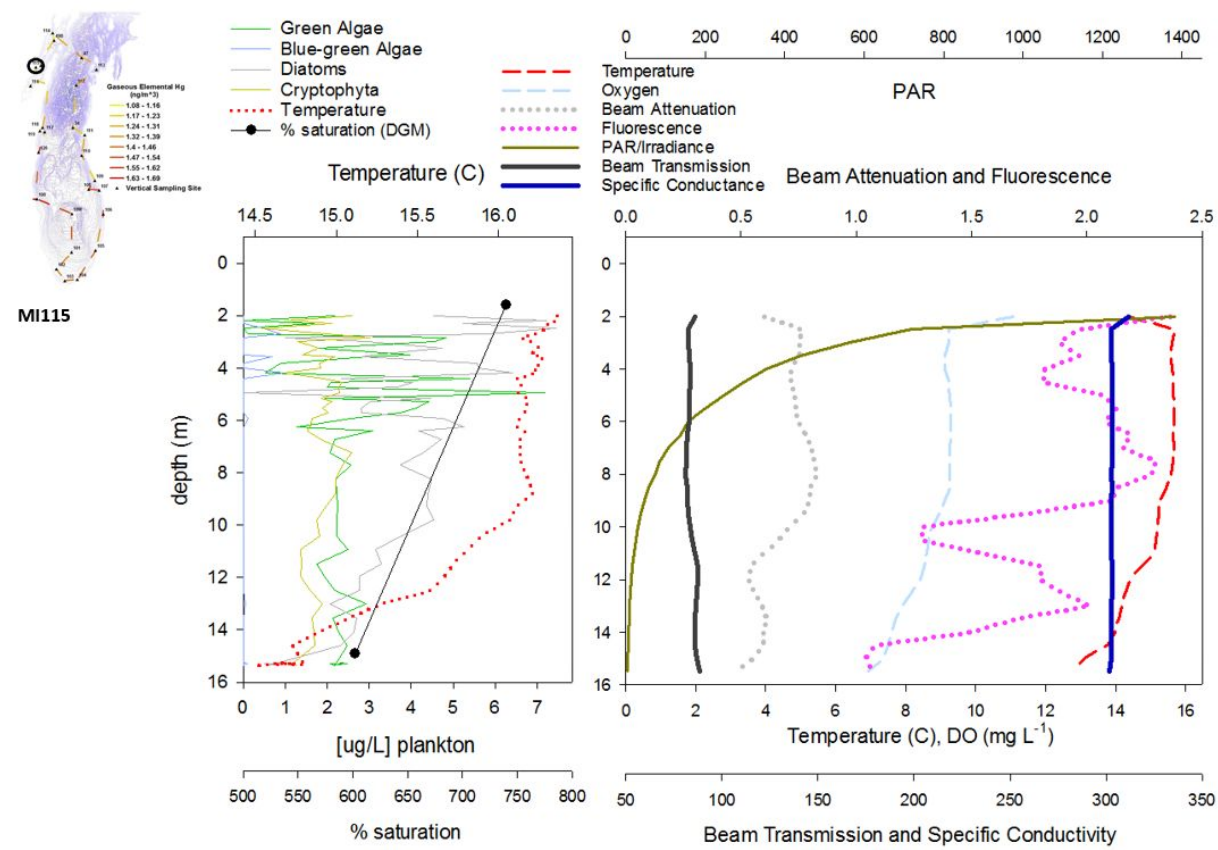

Figure S22: Site - MI116, DGM saturation profile, water quality measurements, and biological fluorescence profiles from the U.S. Environmental Protection Agency's R/V Lake Guardian. PAR is the photosynthetically active radiation. Profile data may be found on the EPA Central Data Exchange.
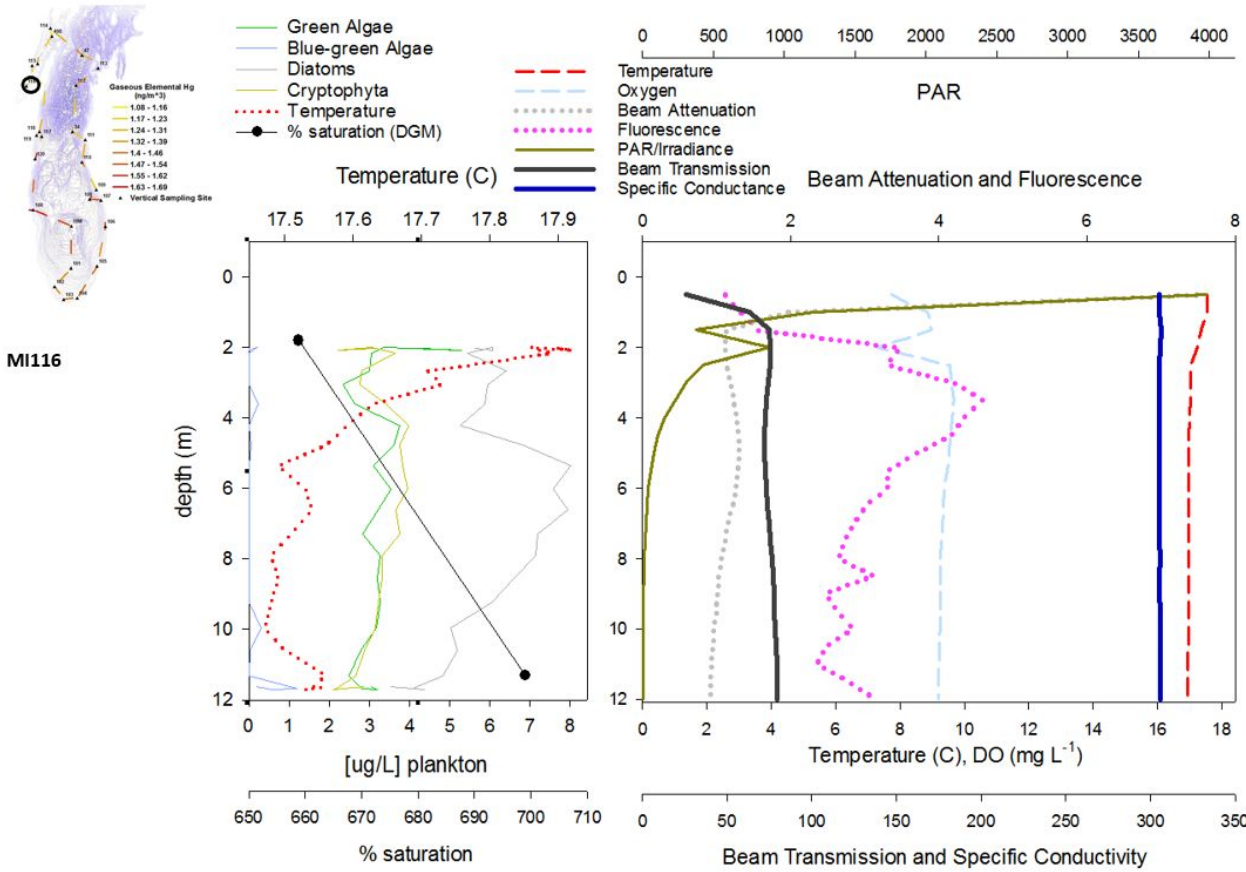
Figure S23: Site - MI117, DGM saturation profile, water quality measurements, and biological fluorescence profiles from the U.S. Environmental Protection Agency's R/V Lake Guardian. PAR is the photosynthetically active radiation. Profile data may be found on the EPA Central Data Exchange.

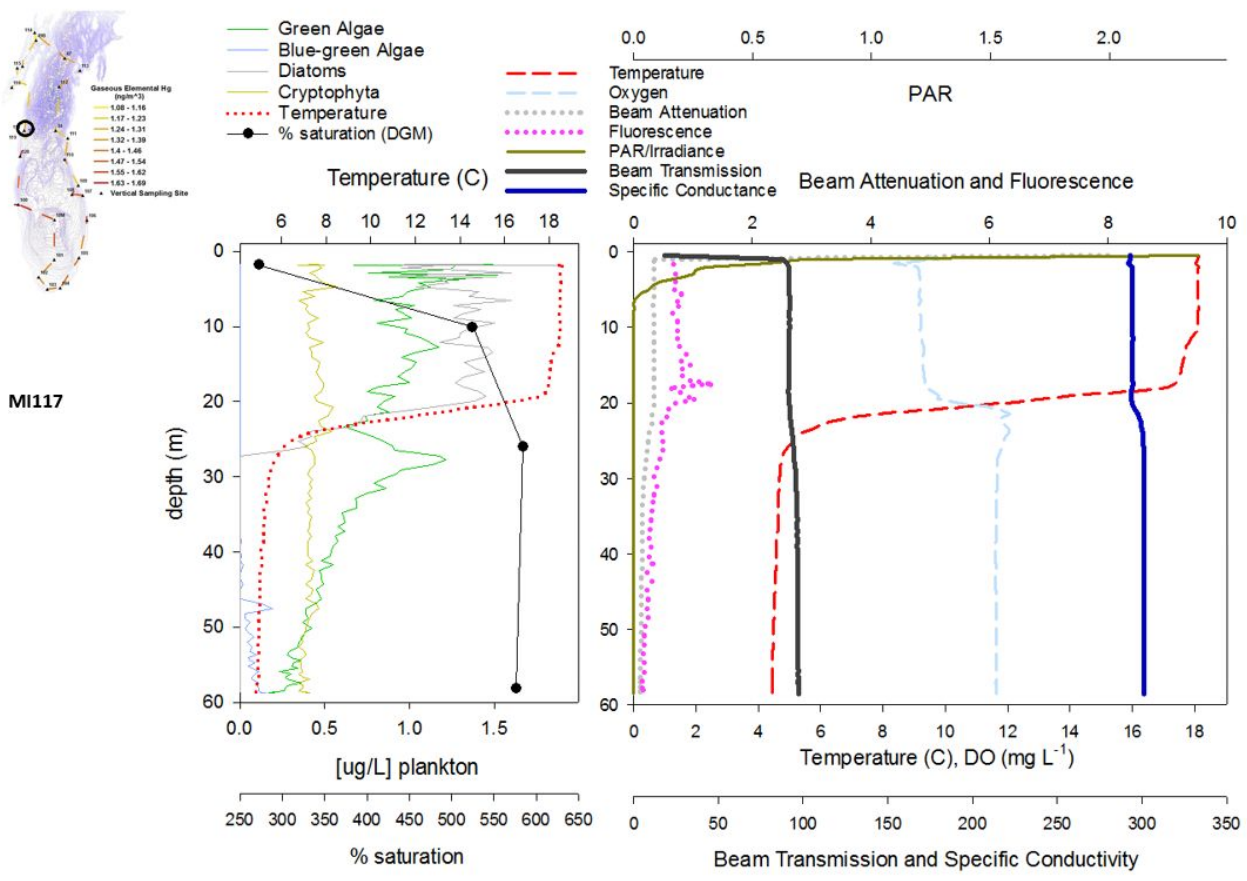

Figure S24: Site - MI118, DGM saturation profile, water quality measurements, and biological fluorescence profiles from the U.S. Environmental Protection Agency's R/V Lake Guardian. PAR is the photosynthetically active radiation. Profile data may be found on the EPA Central Data Exchange.

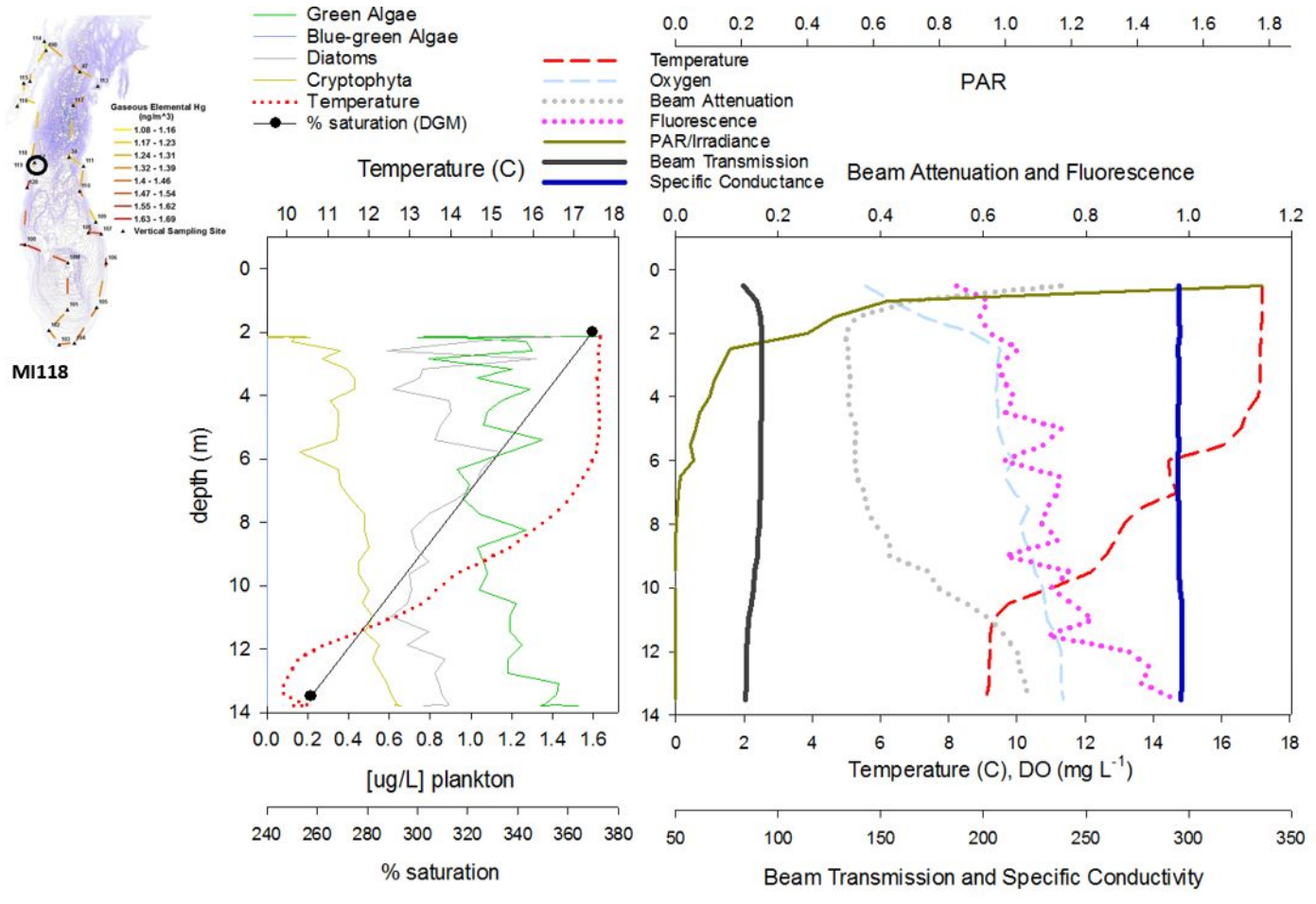


Figure S25: Site - MI107, DGM saturation profile, water quality measurements, and biological fluorescence profiles from the U.S. Environmental Protection Agency's R/V Lake Guardian. PAR is the photosynthetically active radiation. Profile data may be found on the EPA Central Data Exchange.

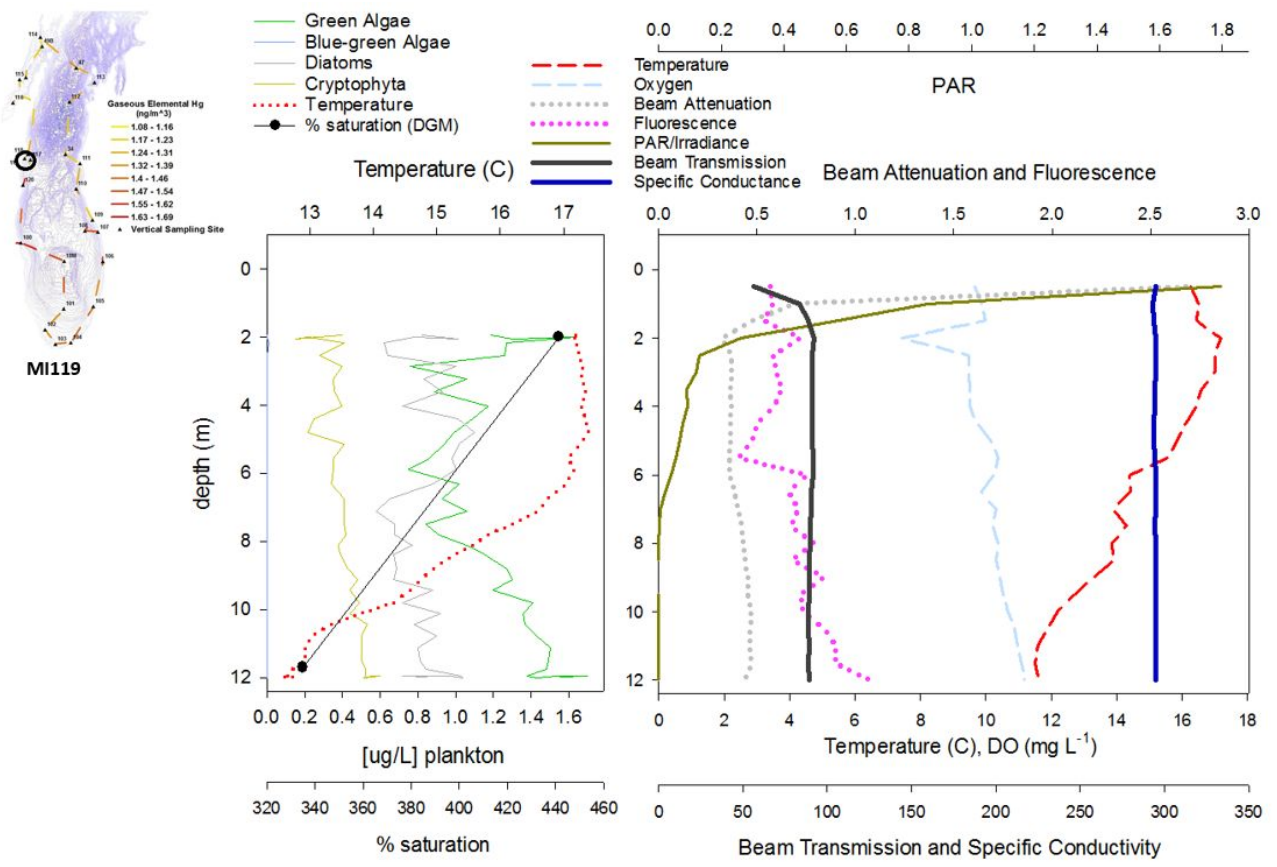

Figure S26: Site - MI120, DGM saturation profile, water quality measurements, and biological fluorescence profiles from the U.S. Environmental Protection Agency's R/V Lake Guardian. PAR is the photosynthetically active radiation. Profile data may be found on the EPA Central Data Exchange.

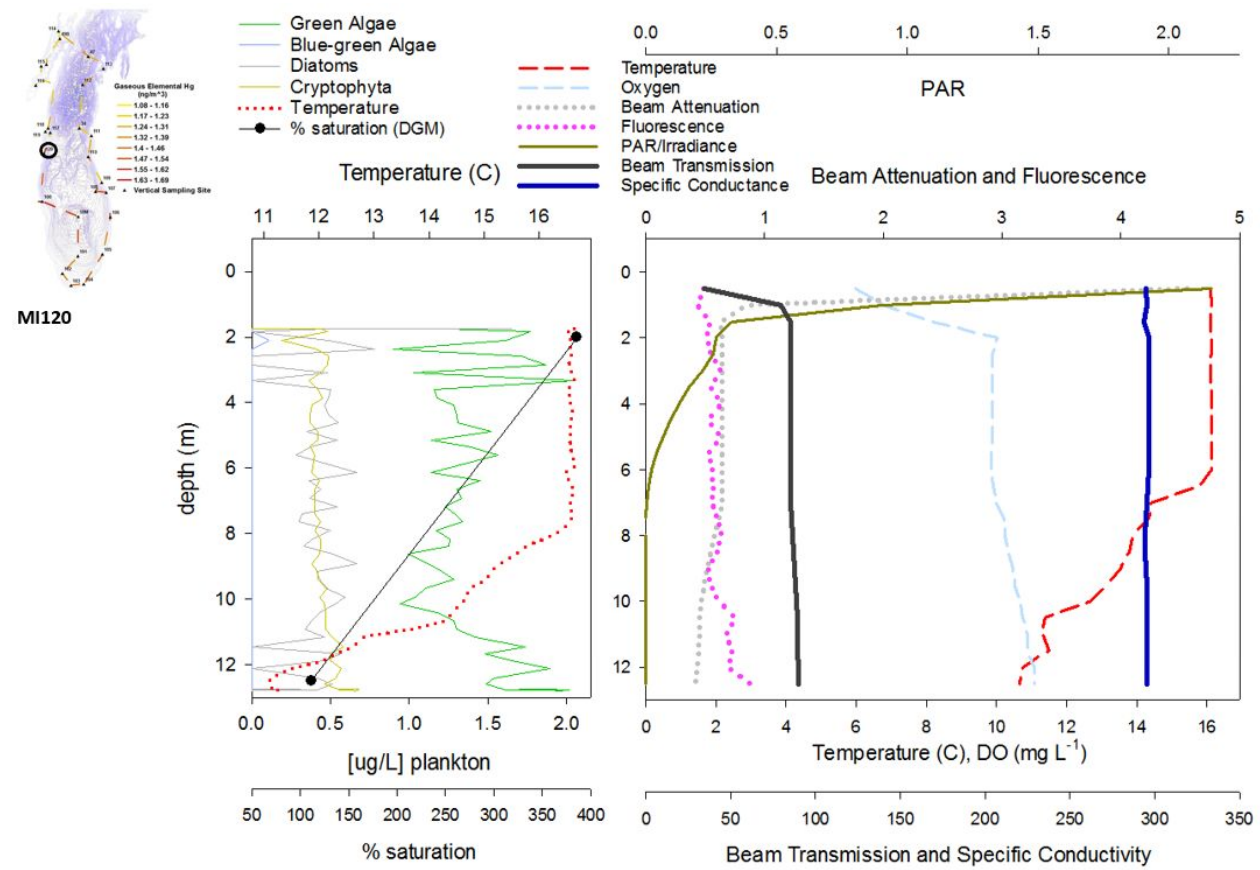


Figure S27: Great Lakes MODIS Imagery for the dates of the Lake Michigan cruise. Data supplied by the NOAA Great Lakes Environmental Research Laboratory, Coast Watch program.

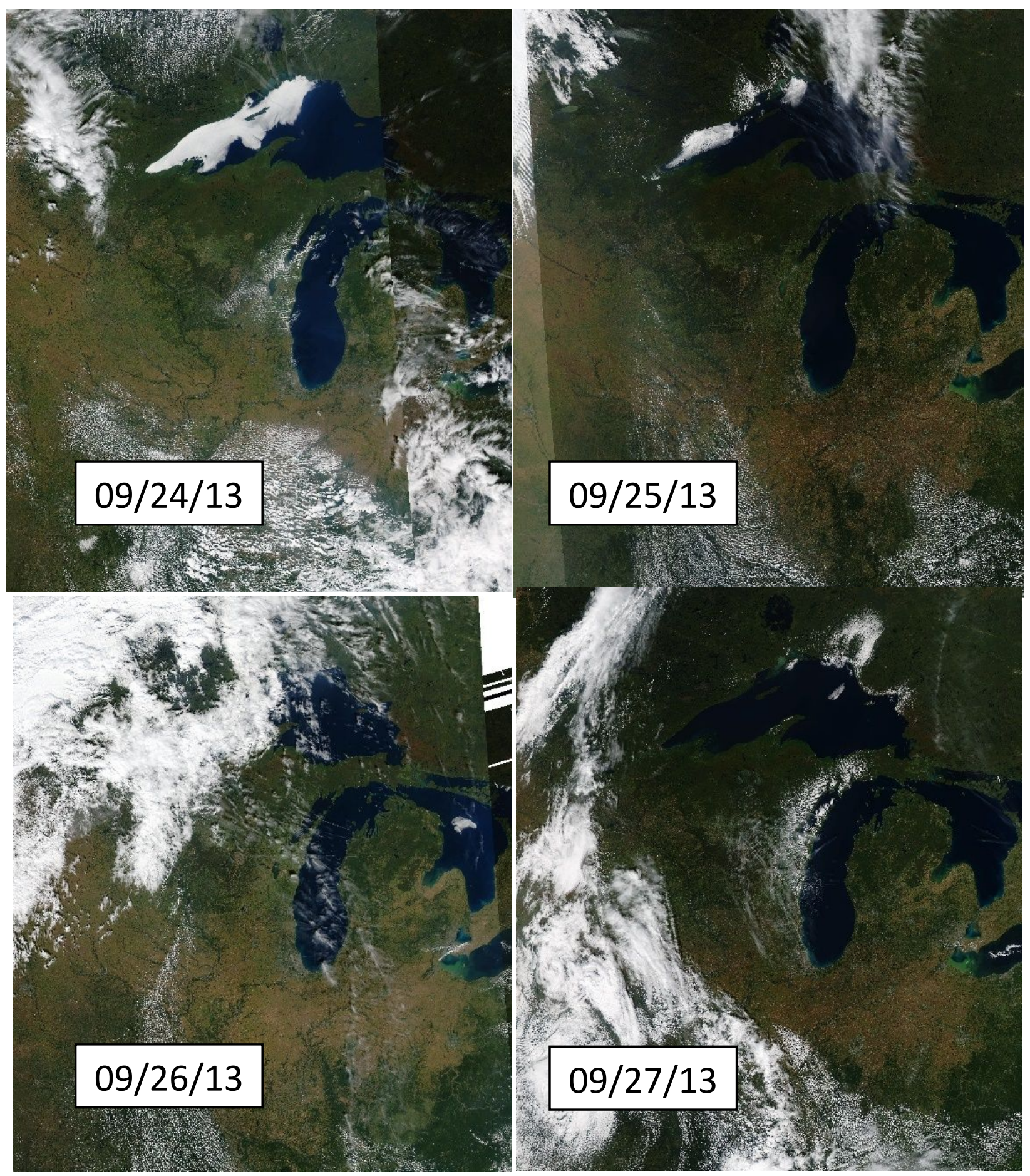


Table S1: Continuous DGM data collection, water temperature and Henry's constant.

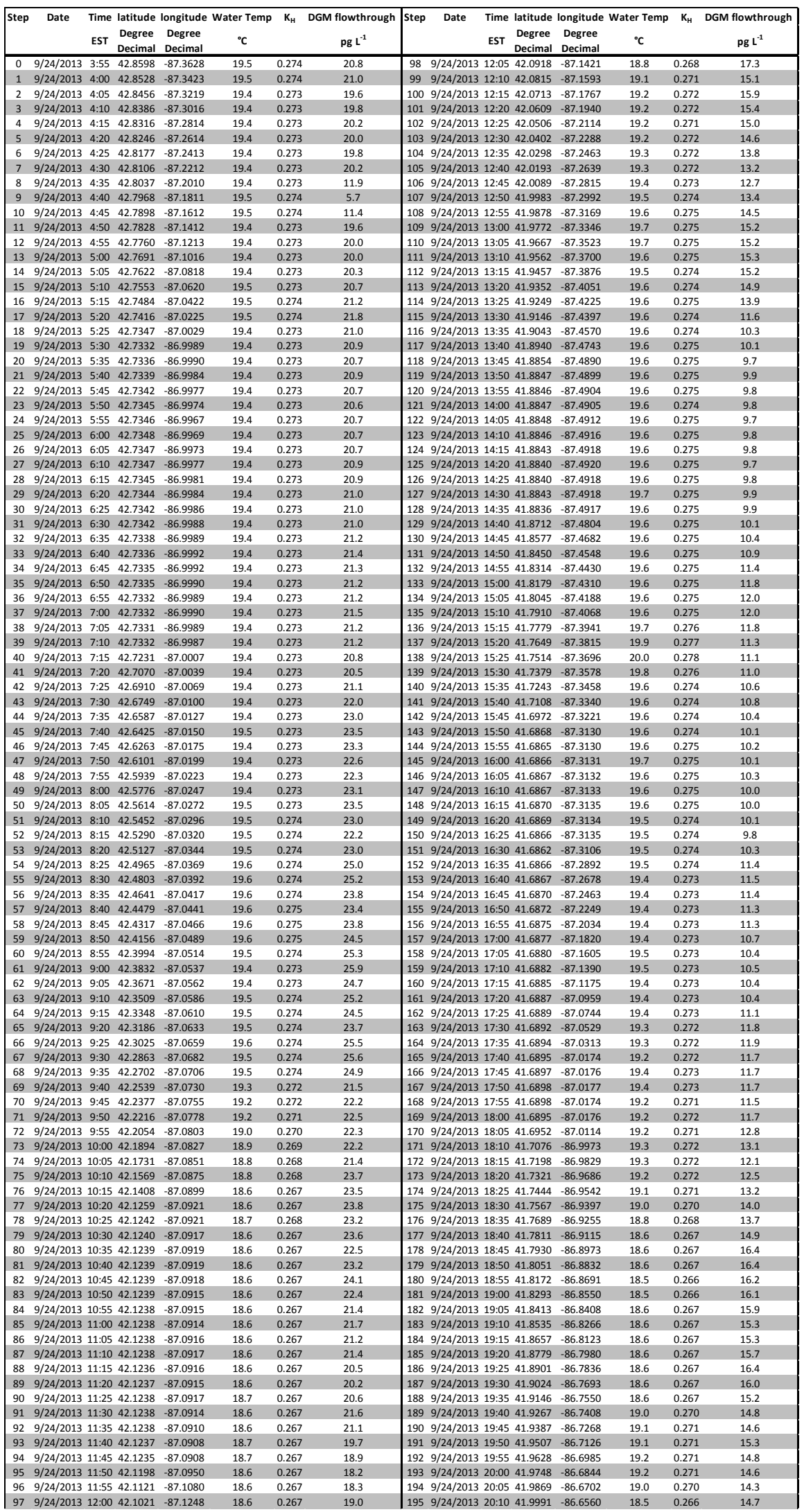


Table S1: continued

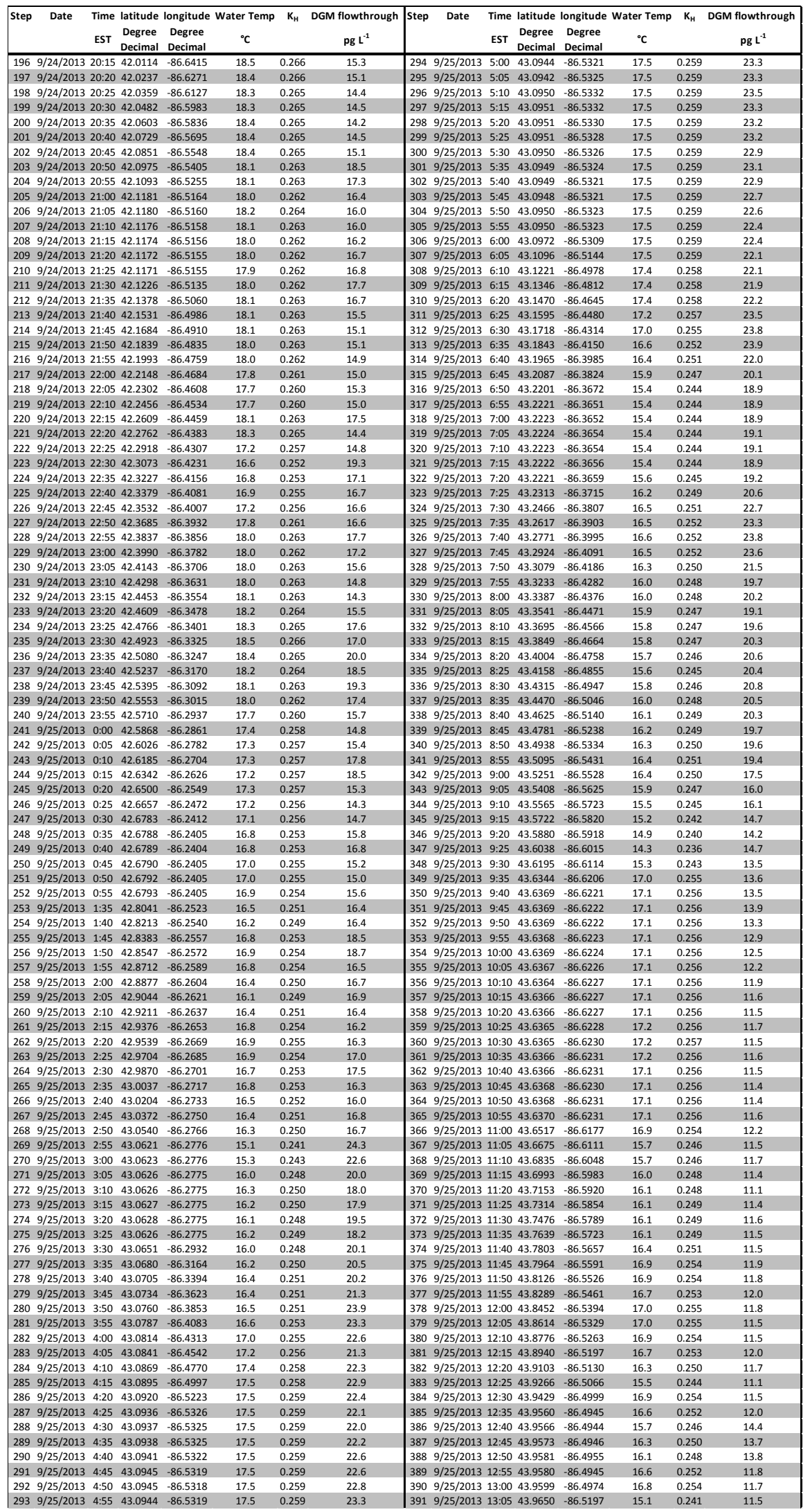


Table S1: continued

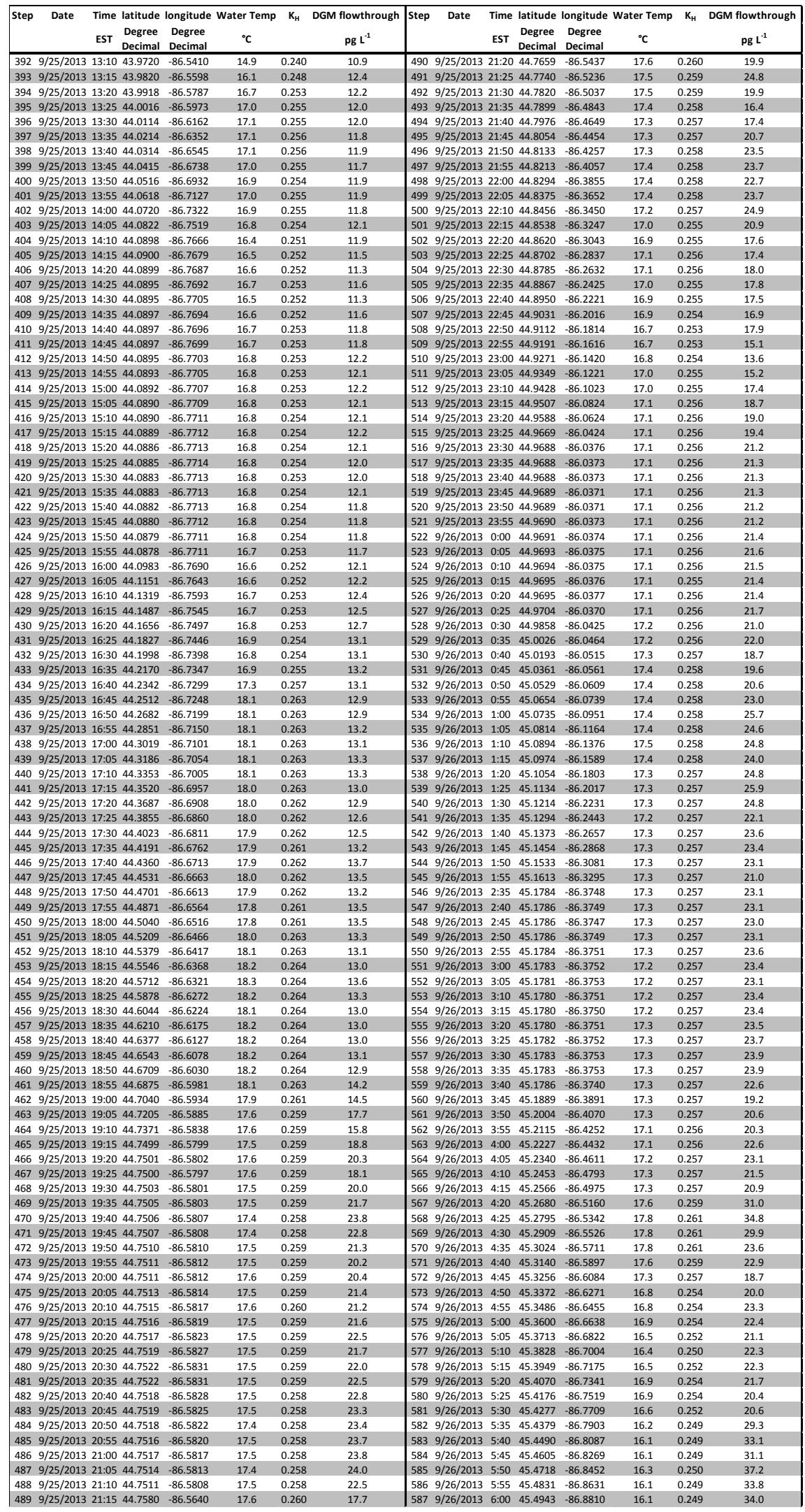


Table S1: continued

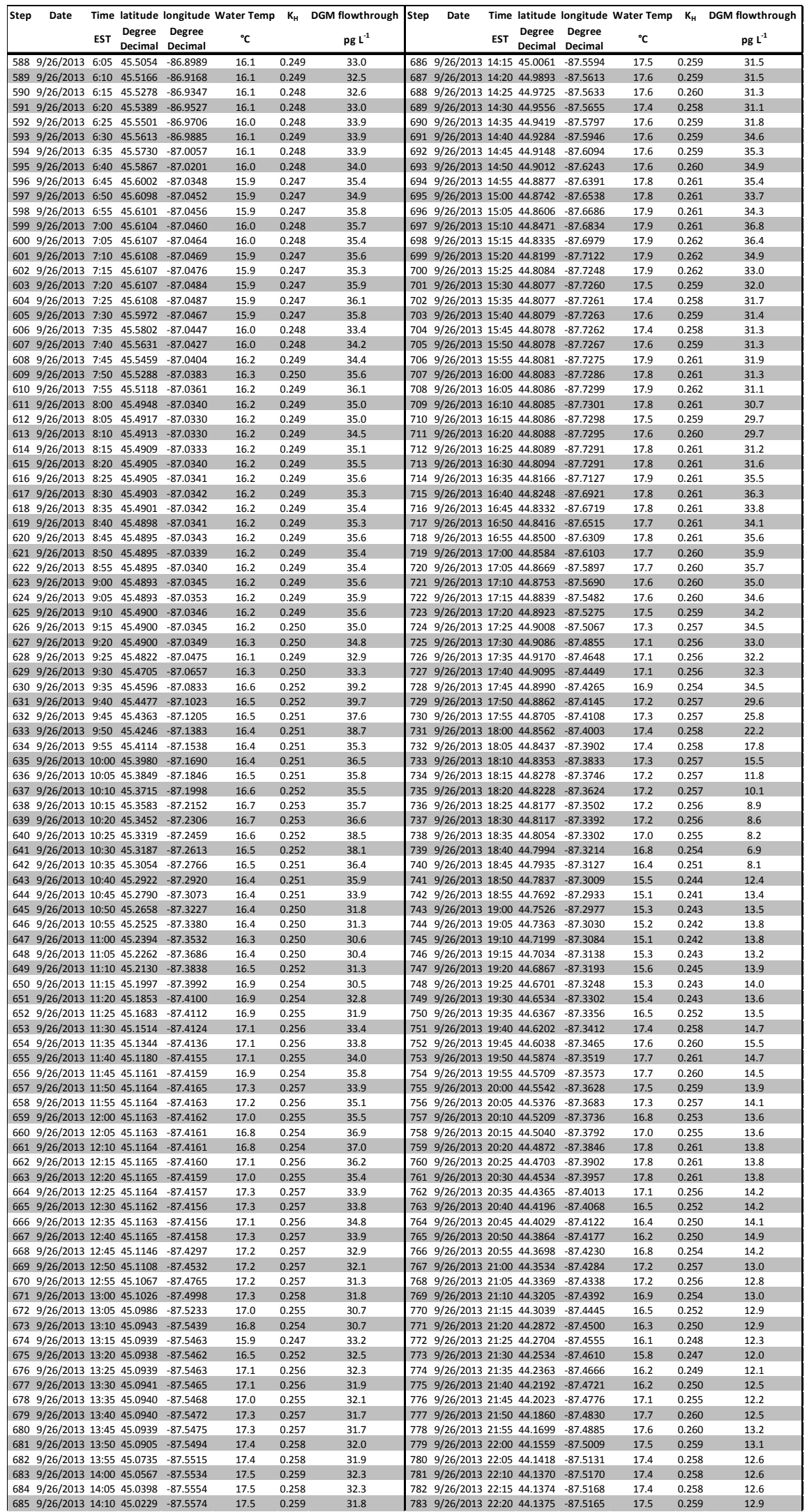


Table S1: continued

\begin{tabular}{|c|c|c|c|c|c|c|c|c|c|c|c|c|c|c|c|}
\hline \multirow[t]{2}{*}{ Step } & \multirow[t]{2}{*}{ Date } & \multirow{2}{*}{$\begin{array}{l}\text { Time } \\
\text { EST }\end{array}$} & \multicolumn{3}{|c|}{ e latitude longitude Water Temp } & \multirow[t]{2}{*}{$\mathrm{K}_{\mathrm{H}}$} & \multirow{2}{*}{$\begin{array}{c}\text { DGM flowthrough } \\
\operatorname{pg~L}^{-1}\end{array}$} & \multirow{2}{*}{ Step } & \multirow[t]{2}{*}{ Date } & \multirow{2}{*}{$\begin{array}{l}\text { Time } \\
\text { EST }\end{array}$} & \multicolumn{3}{|c|}{ latitude longitude Water Temp } & $\mathrm{K}_{\mathrm{H}}$ & DGM flowthrough \\
\hline & & & $\begin{array}{l}\text { Degree } \\
\text { Decimal }\end{array}$ & $\begin{array}{c}\text { Degree } \\
\text { Decimal }\end{array}$ & ${ }^{\circ} \mathrm{C}$ & & & & & & $\begin{array}{l}\text { Degree } \\
\text { Decimal }\end{array}$ & $\begin{array}{c}\text { Degree } \\
\text { Decimal }\end{array}$ & ${ }^{\circ} \mathrm{C}$ & & $\operatorname{pg~L^{-1}}$ \\
\hline 784 & 9/26/2013 & $22: 25$ & 44.1374 & -87.5162 & 17.3 & 0.258 & 13.8 & 844 & 9/27/2013 & $4: 00$ & 43.7476 & $\begin{array}{c}-87.6698 \\
\end{array}$ & 16.3 & 0.250 & 11.0 \\
\hline 785 & 9/26/2013 & $22: 30$ & 44.1373 & -87.5162 & 17.1 & 0.256 & 14.6 & 845 & 9/27/2013 & $4: 05$ & 43.7478 & -87.6696 & 16.3 & 0.250 & 10.9 \\
\hline 786 & 9/26/2013 & $22: 35$ & 44.1375 & -87.5162 & 17.3 & 0.257 & 13.1 & 846 & 9/27/2013 & $4: 10$ & 43.7479 & -87.6694 & 16.3 & 0.250 & 10.8 \\
\hline 787 & 9/26/2013 & 22:40 & 44.1377 & -87.5160 & 17.3 & 0.257 & 12.8 & 847 & 9/27/2013 & $4: 15$ & 43.7480 & -87.6693 & 16.3 & 0.250 & 10.7 \\
\hline 788 & $9 / 26 / 2013$ & 22:45 & 44.1377 & -87.5165 & 17.3 & 0.257 & 13.0 & 848 & 9/27/2013 & $4: 20$ & 43.7481 & -87.6691 & 16.3 & 0.250 & 10.6 \\
\hline 789 & $9 / 26 / 2013$ & $22: 50$ & 44.1376 & -87.5170 & 17.3 & 0.257 & 13.0 & 849 & 9/27/2013 & $4: 25$ & 43.7482 & -87.6689 & 16.3 & 0.250 & 10.7 \\
\hline 790 & $9 / 26 / 2013$ & 22:55 & 44.1377 & -87.5174 & 17.3 & 0.257 & 12.9 & 850 & 9/27/2013 & $4: 30$ & 43.7407 & -87.6708 & 16.2 & 0.249 & 11.7 \\
\hline 791 & $9 / 26 / 2013$ & $23: 00$ & 44.1376 & -87.5179 & 17.4 & 0.258 & 12.9 & 851 & 9/27/2013 & $4: 35$ & 43.7244 & -87.6750 & 17.4 & 0.258 & 14.3 \\
\hline 792 & 9/26/2013 & 23:05 & 44.1258 & -87.5299 & 17.5 & 0.259 & 12.9 & 852 & 9/27/2013 & $4: 40$ & 43.7103 & -87.6782 & 18.1 & 0.263 & 17.6 \\
\hline 793 & 9/26/2013 & $23: 10$ & 44.1146 & -87.5465 & 17.5 & 0.259 & 12.7 & 853 & 9/27/2013 & $4: 45$ & 43.6964 & -87.6819 & 18.1 & 0.264 & 17.6 \\
\hline 794 & 9/26/2013 & $23: 15$ & 44.1031 & -87.5629 & 17.4 & 0.258 & 12.9 & 854 & 9/27/2013 & $4: 50$ & 43.6825 & -87.6852 & 18.1 & 0.263 & 16.8 \\
\hline 795 & $9 / 26 / 2013$ & $23: 20$ & 44.0918 & -87.5794 & 17.2 & 0.257 & 13.1 & 855 & 9/27/2013 & $4: 55$ & 43.6687 & -87.6886 & 18.4 & 0.265 & 18.4 \\
\hline 796 & 9/26/2013 & $23: 25$ & 44.0853 & -87.5887 & 17.0 & 0.255 & 13.2 & 856 & 9/27/2013 & $5: 00$ & 43.6549 & -87.6919 & 18.4 & 0.265 & 19.1 \\
\hline 797 & $9 / 26 / 2013$ & $23: 30$ & 44.0854 & -87.5888 & 17.2 & 0.256 & 13.0 & 857 & 9/27/2013 & $5: 05$ & 43.6410 & -87.6956 & 18.3 & 0.265 & 19.3 \\
\hline 798 & $9 / 26 / 2013$ & 23:35 & 44.0856 & -87.5888 & 17.3 & 0.257 & 13.0 & 858 & 9/27/2013 & $5: 10$ & 43.6269 & -87.6989 & 18.2 & 0.264 & 18.1 \\
\hline 799 & $9 / 26 / 2013$ & $23: 40$ & 44.0856 & -87.5889 & 17.3 & 0.257 & 12.9 & 859 & $9 / 27 / 2013$ & $5: 15$ & 43.6126 & -87.7025 & 18.2 & 0.264 & 17.5 \\
\hline 800 & 9/26/2013 & $23: 45$ & 44.0856 & -87.5890 & 17.3 & 0.257 & 12.8 & 860 & 9/27/2013 & $5: 20$ & 43.5983 & -87.7060 & 18.0 & 0.263 & 16.3 \\
\hline 801 & $9 / 26 / 2013$ & $23: 50$ & 44.0856 & -87.5893 & 17.1 & 0.256 & 12.8 & 861 & 9/27/2013 & $5: 25$ & 43.5840 & -87.7095 & 18.0 & 0.263 & 15.5 \\
\hline 802 & 9/26/2013 & 23:55 & 44.0858 & -87.5894 & 17.2 & 0.257 & 12.8 & 862 & 9/27/2013 & $5: 30$ & 43.5696 & -87.7131 & 18.1 & 0.263 & 15.6 \\
\hline 803 & $9 / 27 / 2013$ & $0: 00$ & 44.0859 & -87.5895 & 17.2 & 0.257 & 12.8 & 863 & 9/27/2013 & $5: 35$ & 43.5552 & -87.7166 & 18.2 & 0.264 & 16.3 \\
\hline 804 & $9 / 27 / 2013$ & $0: 05$ & 44.0862 & -87.5888 & 17.2 & 0.257 & 13.0 & 864 & 9/27/2013 & $5: 40$ & 43.5408 & -87.7201 & 18.2 & 0.264 & 16.3 \\
\hline 805 & $9 / 27 / 2013$ & $0: 10$ & 44.0859 & -87.5885 & 17.2 & 0.257 & 13.0 & 865 & 9/27/2013 & $5: 45$ & 43.5265 & -87.7237 & 18.2 & 0.264 & 15.8 \\
\hline 806 & $9 / 27 / 2013$ & $0: 15$ & 44.0853 & -87.5889 & 17.3 & 0.257 & 12.7 & 866 & 9/27/2013 & $5: 50$ & 43.5122 & -87.7272 & 18.2 & 0.264 & 15.3 \\
\hline 807 & $9 / 27 / 2013$ & $0: 20$ & 44.0849 & -87.5893 & 17.3 & 0.257 & 12.8 & 867 & $9 / 27 / 2013$ & $5: 55$ & 43.4980 & -87.7309 & 18.3 & 0.265 & 15.5 \\
\hline 808 & $9 / 27 / 2013$ & $0: 25$ & 44.0844 & -87.5897 & 17.2 & 0.257 & 13.0 & 868 & 9/27/2013 & $6: 00$ & 43.4840 & -87.7342 & 18.4 & 0.265 & 16.3 \\
\hline 809 & $9 / 27 / 2013$ & $0: 30$ & 44.0826 & -87.5788 & 17.4 & 0.258 & 15.0 & 869 & 9/27/2013 & $6: 05$ & 43.4702 & -87.7377 & 18.6 & 0.267 & 18.1 \\
\hline 810 & $9 / 27 / 2013$ & $0: 35$ & 44.0785 & -87.5561 & 18.0 & 0.262 & 15.9 & 870 & 9/27/2013 & $6: 10$ & 43.4564 & -87.7410 & 18.8 & 0.269 & 20.7 \\
\hline 811 & $9 / 27 / 2013$ & $0: 40$ & 44.0746 & -87.5330 & 18.2 & 0.264 & 17.0 & 871 & $9 / 27 / 2013$ & $6: 15$ & 43.4425 & -87.7445 & 18.9 & 0.269 & 21.8 \\
\hline 812 & 9/27/2013 & $0: 45$ & 44.0706 & -87.5099 & 18.3 & 0.265 & 16.5 & 872 & 9/27/2013 & $6: 20$ & 43.4286 & -87.7478 & 19.0 & 0.270 & 21.1 \\
\hline 813 & 9/27/2013 & $0: 50$ & 44.0667 & -87.4869 & 18.3 & 0.265 & 15.5 & 873 & 9/27/2013 & $6: 25$ & 43.4147 & -87.7512 & 19.0 & 0.270 & 20.5 \\
\hline 814 & 9/27/2013 & $0: 55$ & 44.0646 & -87.4735 & 18.3 & 0.265 & 15.6 & 874 & 9/27/2013 & $6: 30$ & 43.4007 & -87.7546 & 19.0 & 0.270 & 19.8 \\
\hline 815 & $9 / 27 / 2013$ & $1: 00$ & 44.0650 & -87.4728 & 18.3 & 0.265 & 15.6 & 875 & 9/27/2013 & $6: 35$ & 43.3866 & -87.7579 & 19.0 & 0.270 & 19.3 \\
\hline 816 & $9 / 27 / 2013$ & 1:05 & 44.0655 & -87.4728 & 18.2 & 0.264 & 17.1 & 876 & 9/27/2013 & $6: 40$ & 43.3727 & -87.7615 & 19.0 & 0.270 & 18.9 \\
\hline 817 & $9 / 27 / 2013$ & $1: 10$ & 44.0659 & -87.4726 & 18.2 & 0.264 & 17.8 & 877 & 9/27/2013 & $6: 45$ & 43.3587 & -87.7651 & 19.0 & 0.270 & 19.0 \\
\hline 818 & $9 / 27 / 2013$ & $1: 15$ & 44.0663 & -87.4723 & 18.3 & 0.265 & 15.5 & 878 & 9/27/2013 & $6: 50$ & 43.3446 & -87.7682 & 19.0 & 0.270 & 19.5 \\
\hline 819 & $9 / 27 / 2013$ & $1: 20$ & 44.0664 & -87.4718 & 18.3 & 0.265 & 15.6 & 879 & 9/27/2013 & $6: 55$ & 43.3306 & -87.7718 & 19.1 & 0.271 & 20.0 \\
\hline 820 & $9 / 27 / 2013$ & $1: 25$ & 44.0668 & -87.4727 & 18.3 & 0.265 & 15.7 & 880 & 9/27/2013 & $7: 00$ & 43.3165 & -87.7752 & 19.1 & 0.271 & 21.0 \\
\hline 821 & $9 / 27 / 2013$ & $1: 30$ & 44.0678 & -87.4720 & 18.3 & 0.265 & 15.6 & 881 & $9 / 27 / 2013$ & $7: 05$ & 43.3023 & -87.7787 & 19.1 & 0.271 & 21.4 \\
\hline 822 & 9/27/2013 & $1: 35$ & 44.0679 & -87.4717 & 18.3 & 0.265 & 15.6 & 882 & 9/27/2013 & $7: 10$ & 43.2881 & -87.7822 & 18.9 & 0.269 & 21.8 \\
\hline 823 & $9 / 27 / 2013$ & $1: 40$ & 44.0684 & -87.4716 & 18.3 & 0.265 & 15.6 & 883 & 9/27/2013 & $7: 15$ & 43.2739 & -87.7856 & 18.6 & 0.267 & 20.0 \\
\hline 824 & 9/27/2013 & $1: 45$ & 44.0686 & -87.4715 & 18.3 & 0.265 & 15.6 & 884 & 9/27/2013 & $7: 20$ & 43.2597 & -87.7890 & 18.6 & 0.267 & 17.7 \\
\hline 825 & $9 / 27 / 2013$ & $1: 50$ & 44.0686 & -87.4716 & 18.3 & 0.265 & 15.7 & 885 & 9/27/2013 & $7: 25$ & 43.2456 & -87.7925 & 18.6 & 0.267 & 18.3 \\
\hline 826 & $9 / 27 / 2013$ & $1: 55$ & 44.0681 & -87.4723 & 18.3 & 0.265 & 15.8 & 886 & 9/27/2013 & $7: 30$ & 43.2315 & -87.7960 & 18.5 & 0.266 & 19.9 \\
\hline 827 & $9 / 27 / 2013$ & $2: 00$ & 44.0531 & -87.4813 & 18.3 & 0.265 & 16.2 & 887 & 9/27/2013 & $7: 35$ & 43.2172 & -87.7994 & 18.4 & 0.265 & 18.3 \\
\hline 828 & $9 / 27 / 2013$ & 2:05 & 44.0379 & -87.4912 & 18.3 & 0.265 & 17.5 & 888 & 9/27/2013 & $7: 40$ & 43.2029 & -87.8030 & 18.4 & 0.265 & 17.1 \\
\hline 829 & $9 / 27 / 2013$ & $2: 10$ & 44.0224 & -87.5005 & 18.3 & 0.265 & 17.3 & 889 & 9/27/2013 & $7: 45$ & 43.1885 & -87.8064 & 18.5 & 0.266 & 17.2 \\
\hline 830 & $9 / 27 / 2013$ & $2: 15$ & 44.0070 & -87.5102 & 18.2 & 0.264 & 17.1 & 890 & 9/27/2013 & $7: 50$ & 43.1741 & -87.8101 & 18.4 & 0.266 & 17.1 \\
\hline 831 & 9/27/2013 & $2: 20$ & 43.9916 & -87.5197 & 18.2 & 0.264 & 19.5 & 891 & $9 / 27 / 2013$ & $7: 55$ & 43.1598 & -87.8135 & 18.4 & 0.265 & 15.6 \\
\hline 832 & 9/27/2013 & $2: 25$ & 43.9763 & -87.5292 & 18.3 & 0.2 & 18.5 & 892 & 9/27/2013 & $8: 00$ & 43.1454 & -87.8171 & 18.4 & 0.266 & 15.3 \\
\hline 833 & $9 / 27 / 2013$ & $2: 30$ & 43.9611 & -87.5387 & 18.2 & 0.264 & 20.4 & 893 & $9 / 27 / 2013$ & $8: 05$ & 43.1310 & -87.8205 & 18.4 & 0.266 & 14.5 \\
\hline 834 & 9/27/2013 & $2: 35$ & 43.9456 & -87.5481 & 18.2 & 0.264 & 17.1 & 894 & 9/27/2013 & $8: 10$ & 43.1166 & -87.8241 & 18.4 & 0.266 & 14.1 \\
\hline 835 & $9 / 27 / 2013$ & $2: 40$ & 43.9305 & -87.5577 & 18.3 & 0.265 & 18.2 & 895 & 9/27/2013 & $8: 15$ & 43.1022 & -87.8276 & 18.5 & 0.266 & 13.0 \\
\hline 836 & 9/27/2013 & $2: 45$ & 43.9154 & -87.5669 & 18.4 & 0.266 & 17.5 & 896 & 9/27/2013 & $8: 20$ & 43.0878 & -87.8311 & 18.5 & 0.266 & 11.7 \\
\hline 837 & $9 / 27 / 2013$ & $2: 50$ & 43.9004 & -87.5763 & 18.4 & 0.266 & 15.8 & 897 & 9/27/2013 & $8: 25$ & 43.0734 & -87.8346 & 18.5 & 0.266 & 14.7 \\
\hline 838 & $9 / 27 / 2013$ & $2: 55$ & 43.8853 & -87.5856 & 18.2 & 0.264 & 15.4 & 898 & 9/27/2013 & $8: 30$ & 43.0591 & -87.8381 & 18.4 & 0.265 & 16.2 \\
\hline 839 & $9 / 27 / 2013$ & $3: 35$ & 43.7617 & -87.6620 & 16.5 & 0.252 & 14.4 & 899 & 9/27/2013 & $8: 35$ & 43.0483 & -87.8508 & 18.4 & 0.265 & 14.1 \\
\hline 840 & $9 / 27 / 2013$ & $3: 40$ & 43.7483 & -87.6700 & 16.3 & 0.250 & 11.1 & 900 & 9/27/2013 & $8: 40$ & 43.0380 & -87.8648 & 18.3 & 0.265 & 14.0 \\
\hline 841 & $9 / 27 / 2013$ & $3: 45$ & 43.7478 & -87.6699 & 16.3 & 0.250 & 10.9 & 901 & $9 / 27 / 2013$ & $8: 45$ & 43.0275 & -87.8786 & 18.3 & 0.265 & 12.0 \\
\hline 842 & 9/27/2013 & $3: 50$ & 43.7476 & -87.6698 & 16.3 & 0.250 & 10.9 & 902 & 9/27/2013 & $8: 50$ & 43.0255 & -87.8915 & 18.8 & 0.268 & 8.0 \\
\hline 843 & $9 / 27 / 2013$ & $3: 55$ & 43.7476 & -87.6698 & 16.3 & 0.250 & 11.0 & & & & & & & & \\
\hline
\end{tabular}


Table S2: Atmospheric Hg measurements (GEM - gaseous elemental Hg, PBM - particle bound Hg, GOM - gaseous oxidized Hg), meteorological data, averaged DGM from the continuous measurements and temperature and wind-dependent terms for determining percent saturation of surface DGM.

\begin{tabular}{|c|c|c|c|c|c|c|c|c|c|c|c|c|c|c|c|}
\hline Date Time & Date Time & GEM & PBM & GOM & $\begin{array}{l}\text { Wind } \\
\text { Speed }\end{array}$ & Water Temp & kH & Ave. DGM (flowthrough) & Diffusivity - $\mathrm{Hg}$ & idt Number & $k_{600}$ & $\mathrm{k}_{\mathrm{u}}$ & $\Delta c^{\text {air-water }}$ & Flux & $\%$ saturation of \\
\hline EST - start & EST - end & $\mathrm{ng} \mathrm{m}^{-3}$ & $\mathrm{pg} \mathrm{m}^{-3}$ & $\mathrm{pg} \mathrm{m}^{-3}$ & $\mathrm{~m} \mathrm{~s}^{-1}$ & ${ }^{\circ} \mathrm{C}$ & & over sequence - $\mathrm{pg} \mathrm{L}^{-3}$ & $10 e^{-5} \mathrm{~cm}^{2} \mathrm{~s}^{-1}$ & (Sc) & $\begin{array}{c}\text { Wind- } \\
\text { dependent Sc }\end{array}$ & $\begin{array}{l}\text { Corrected kinetic } \\
\text { viscosity }\end{array}$ & ${ }^{\circ} \mathrm{C}$ & $\begin{array}{l}\mathrm{pg} \mathrm{m}^{-2} \\
\text { day }^{-1}\end{array}$ & DGM \\
\hline 9/24/13 2:10 & 9/24/13 3:10 & 1.56 & 3.6 & 3.4 & 8.8 & 19.5 & 0.274 & 20.5 & $1.7 \mathrm{E}-05$ & 618 & 20.1 & 20.4 & -19.3 & -3.9 & $366 \%$ \\
\hline 9/24/13 4:10 & 9/24/13 5:10 & 1.48 & 2.8 & 9.6 & 9.4 & 19.4 & 0.273 & 20.1 & $1.6 \mathrm{E}-05$ & 621 & 22.7 & 23.1 & -18.3 & -4.2 & $366 \%$ \\
\hline 9/24/13 18:10 & 9/24/13 19:10 & 1.42 & 5.8 & 5.7 & 4.1 & 19.3 & 0.272 & 14.6 & $1.6 \mathrm{E}-05$ & 626 & 5.0 & 5.1 & -11.7 & -0.6 & $252 \%$ \\
\hline 9/24/13 20:10 & $9 / 24 / 1321: 10$ & 1.44 & 6.1 & 4.3 & 5.5 & 18.5 & 0.266 & 15.5 & $1.6 \mathrm{E}-05$ & 652 & 8.4 & 8.8 & -13.3 & -1.2 & $271 \%$ \\
\hline 9/24/13 22:10 & 9/24/13 23:10 & 1.38 & 5.4 & 18.3 & 2.8 & 17.7 & 0.260 & 16.4 & $1.6 \mathrm{E}-05$ & 680 & 2.7 & 2.8 & -13.7 & -0.4 & $284 \%$ \\
\hline 9/25/13 0:10 & 9/25/13 1:10 & 1.50 & 9.0 & 7.0 & 2.8 & 17.3 & 0.257 & 16.5 & $1.6 \mathrm{E}-05$ & 694 & 2.7 & 2.9 & -16.3 & -0.5 & $306 \%$ \\
\hline 9/25/13 3:10 & 9/25/13 4:10 & 1.49 & 3.2 & 3.0 & 2.8 & 16.3 & 0.250 & 20.7 & $1.5 \mathrm{E}-05$ & 727 & 2.7 & 2.9 & -16.6 & -0.5 & $304 \%$ \\
\hline 9/25/13 5:10 & 9/25/136:10 & 1.49 & 5.1 & 9.8 & 5.6 & 17.5 & 0.259 & 22.8 & $1.6 \mathrm{E}-05$ & 686 & 8.8 & 9.4 & -22.0 & -2.1 & $408 \%$ \\
\hline 9/25/13 19:10 & 9/25/13 20:10 & 1.23 & 0.0 & 10.4 & 3.0 & 17.6 & 0.259 & 20.4 & $1.6 \mathrm{E}-05$ & 683 & 3.0 & 3.2 & -14.6 & -0.5 & $335 \%$ \\
\hline 9/25/13 21:10 & 9/25/13 22:10 & 1.29 & 9.5 & 7.2 & 2.0 & 17.5 & 0.258 & 21.4 & $1.6 \mathrm{E}-05$ & 687 & 1.6 & 1.7 & -21.2 & -0.4 & $452 \%$ \\
\hline 9/25/13 23:10 & 9/26/130:10 & 1.25 & 2.8 & 6.7 & 3.0 & 17.0 & 0.255 & 20.5 & $1.6 \mathrm{E}-05$ & 702 & 3.0 & 3.2 & -16.2 & -0.5 & $356 \%$ \\
\hline 9/26/13 1:10 & 9/26/13 2:10 & 1.27 & 1.8 & 5.2 & 4.0 & 17.5 & 0.258 & 23.7 & $1.6 \mathrm{E}-05$ & 688 & 4.9 & 5.2 & -23.5 & -1.2 & $504 \%$ \\
\hline 9/26/13 3:10 & 9/26/13 4:10 & 1.38 & 1.3 & 4.9 & 4.0 & 17.2 & 0.257 & 22.5 & $1.6 \mathrm{E}-05$ & 695 & 4.9 & 5.3 & -22.0 & -1.2 & $436 \%$ \\
\hline 9/26/13 19:10 & $9 / 26 / 1320: 10$ & 1.22 & 6.7 & 9.5 & 4.3 & 15.1 & 0.242 & 14.1 & $1.5 \mathrm{E}-05$ & 767 & 5.5 & 6.3 & -12.5 & -0.8 & $273 \%$ \\
\hline 9/26/13 21:10 & 9/26/13 22:10 & 1.25 & 8.1 & 15.4 & 4.0 & 16.9 & 0.254 & 12.6 & $1.6 \mathrm{E}-05$ & 707 & 4.9 & 5.3 & -11.7 & -0.6 & $264 \%$ \\
\hline 9/26/13 23:10 & 9/27/130:10 & 1.21 & 6.2 & 26.4 & 3.5 & 17.5 & 0.259 & 12.9 & $1.6 \mathrm{E}-05$ & 685 & 3.9 & 4.2 & -11.5 & -0.5 & $272 \%$ \\
\hline 9/27/13 1:10 & 9/27/13 2:10 & 1.20 & 4.3 & 10.3 & 4.4 & 18.2 & 0.264 & 16.1 & $1.6 \mathrm{E}-05$ & 662 & 5.8 & 6.1 & -16.6 & -1.0 & $393 \%$ \\
\hline 9/27/13 3:10 & $9 / 27 / 134: 10$ & 1.61 & 20.9 & 5.5 & 1.2 & 16.5 & 0.252 & 11.4 & $1.5 \mathrm{E}-05$ & 719 & 0.7 & 0.8 & -12.8 & -0.1 & $225 \%$ \\
\hline 9/24/13 6:10 & 9/24/13 7:10 & 1.47 & 4.0 & 4.3 & 8.3 & 19.4 & 0.273 & 21.2 & $1.6 \mathrm{E}-05$ & 621 & 18.1 & 18.4 & -19.4 & -3.6 & $387 \%$ \\
\hline 9/27/13 6:10 & $9 / 27 / 137: 10$ & 1.44 & 2.9 & 12.5 & 0.5 & 18.8 & 0.269 & 20.4 & $1.6 \mathrm{E}-05$ & 641 & 0.2 & 0.2 & -19.3 & 0.0 & $386 \%$ \\
\hline 9/25/13 17:10 & 9/25/13 18:10 & 1.19 & 0.0 & 12.6 & 2.4 & 18.1 & 0.263 & 13.2 & $1.6 \mathrm{E}-05$ & 665 & 2.0 & 2.1 & -12.1 & -0.3 & $294 \%$ \\
\hline 9/25/13 7:10 & 9/25/13 8:10 & 1.18 & 3.0 & 3.3 & 5.3 & 15.4 & 0.244 & 20.9 & $1.5 \mathrm{E}-05$ & 757 & 7.9 & 8.8 & -17.9 & -1.6 & $393 \%$ \\
\hline 9/24/13 16:10 & 9/24/13 17:10 & 1.40 & 8.7 & 7.3 & 3.9 & 19.6 & 0.275 & 10.7 & 1.7E-05 & 614 & 4.8 & 4.8 & -8.6 & -0.4 & $198 \%$ \\
\hline $9 / 24 / 138: 10$ & 9/24/13 9:10 & 1.48 & 4.3 & 2.4 & 8.5 & 19.5 & 0.274 & 24.2 & 1.7E-05 & 619 & 18.9 & 19.2 & -21.5 & -4.1 & $425 \%$ \\
\hline 9/24/13 12:10 & 9/24/13 13:10 & 1.36 & 6.6 & 9.7 & 6.9 & 19.1 & 0.271 & 14.6 & $1.6 \mathrm{E}-05$ & 631 & 12.9 & 13.2 & -13.7 & -1.8 & $301 \%$ \\
\hline 9/25/13 15:10 & 9/25/13 16:10 & 1.24 & 2.4 & 13.9 & 1.7 & 16.8 & 0.253 & 12.0 & $1.5 \mathrm{E}-05$ & 710 & 1.2 & 1.3 & -10.9 & -0.1 & $248 \%$ \\
\hline 9/25/139:10 & 9/25/13 10:10 & 1.32 & 4.8 & 10.7 & 3.3 & 15.5 & 0.245 & 13.6 & $1.5 \mathrm{E}-05$ & 753 & 3.4 & 3.8 & -14.8 & -0.6 & $300 \%$ \\
\hline 9/24/13 14:10 & 9/24/13 15:10 & 1.37 & 7.3 & 9.5 & 7.6 & 19.6 & 0.275 & 10.6 & 1.7E-05 & 614 & 15.5 & 15.7 & -8.4 & -1.3 & $196 \%$ \\
\hline 9/24/13 10:10 & 9/24/13 11:10 & 1.42 & 5.4 & 4.4 & 8.1 & 18.8 & 0.268 & 22.7 & $1.6 \mathrm{E}-05$ & 642 & 17.3 & 17.9 & -22.3 & -4.0 & $449 \%$ \\
\hline 9/25/13 13:10 & 9/25/13 14:10 & 1.21 & 5.5 & 12.9 & 4.4 & 14.9 & 0.240 & 11.9 & $1.5 \mathrm{E}-05$ & 773 & 5.8 & 6.6 & -9.7 & -0.6 & $216 \%$ \\
\hline 9/25/13 11:10 & 9/25/13 12:10 & 1.25 & 6.4 & 12.9 & 4.4 & 15.7 & 0.246 & 11.6 & $1.5 \mathrm{E}-05$ & 748 & 5.8 & 6.4 & -10.4 & -0.7 & $229 \%$ \\
\hline 9/26/135:10 & $9 / 26 / 136: 10$ & 1.30 & 1.2 & 3.9 & 3.0 & 16.4 & 0.250 & 28.6 & $1.5 \mathrm{E}-05$ & 725 & 3.0 & 3.3 & -21.0 & -0.7 & $429 \%$ \\
\hline 9/26/13 7:10 & 9/26/13 8:10 & 1.08 & 1.4 & 3.4 & 1.3 & 15.9 & 0.247 & 35.1 & $1.5 \mathrm{E}-05$ & 740 & 0.8 & 0.9 & -34.5 & -0.3 & $813 \%$ \\
\hline 9/26/139:10 & 9/26/13 10:10 & 1.17 & 1.0 & 2.7 & 3.6 & 16.2 & 0.249 & 36.1 & $1.5 \mathrm{E}-05$ & 731 & 4.1 & 4.5 & -34.4 & -1.5 & $760 \%$ \\
\hline 9/26/13 11:10 & 9/26/13 12:10 & 1.25 & 4.3 & 4.4 & 2.8 & 16.5 & 0.252 & 34.0 & $1.5 \mathrm{E}-05$ & 719 & 2.7 & 2.9 & -30.0 & -0.9 & $631 \%$ \\
\hline 9/26/13 13:10 & 9/26/13 14:10 & 1.17 & 6.6 & 5.0 & 1.5 & 16.8 & 0.254 & 32.0 & $1.5 \mathrm{E}-05$ & 710 & 1.0 & 1.1 & -29.6 & -0.3 & $664 \%$ \\
\hline 9/26/13 15:10 & 9/26/13 16:10 & 1.27 & 6.0 & 12.8 & 0.9 & 17.9 & 0.261 & 32.6 & $1.6 \mathrm{E}-05$ & 674 & 0.5 & 0.5 & -35.5 & -0.2 & $756 \%$ \\
\hline 9/26/13 17:10 & 9/26/13 18:10 & 1.18 & 4.4 & 14.9 & 2.5 & 17.6 & 0.260 & 29.3 & $1.6 \mathrm{E}-05$ & 681 & 2.2 & 2.4 & -33.8 & -0.8 & $767 \%$ \\
\hline
\end{tabular}


Table S3: Vertical profile data of filter-passing total mercury (FHgT) and dissolved organic carbon (DOC) concentrations over operationally defined water layers paired to manually-run DGM concentrations of the profile. Depth categories are defined as surface at $2 \mathrm{~m}$ (SRF), mid-epilimnion (MEP), thermocline (TRM), mid-hypolimnion (MHY), and 2 meters from the benthos (B-).

\begin{tabular}{|c|c|c|c|c|c|c|c|c|c|}
\hline Site & Date & $\begin{array}{l}\text { Time } \\
\text { EST }\end{array}$ & $\begin{array}{l}\text { Latitude } \\
\text { Degree } \\
\text { Decimal }\end{array}$ & $\begin{array}{l}\text { Longitude } \\
\text { Degree } \\
\text { Decimal }\end{array}$ & $\begin{array}{l}\text { Depth } \\
\text { category }\end{array}$ & $\begin{array}{l}\text { Depth } \\
\text { meters }\end{array}$ & $\begin{array}{c}\text { DOC } \\
\mathrm{mg} \mathrm{L}^{-1}\end{array}$ & $\begin{array}{l}\text { FHgT } \\
\mathrm{ng} \mathrm{L}^{-1}\end{array}$ & $\begin{array}{c}\text { DGM Manual } \\
\text { pg L }^{-1}\end{array}$ \\
\hline \multirow{3}{*}{ Ml100 } & \multirow{3}{*}{$9 / 24 / 2013$} & \multirow{3}{*}{ 1:19 AM } & \multirow{3}{*}{43.0135} & \multirow{3}{*}{-87.8113} & MEP & 7.0 & 1.85 & 0.192 & 19.8 \\
\hline & & & & & TRM & 14.0 & 1.73 & 0.129 & 20.2 \\
\hline & & & & & B- & 17.2 & 1.88 & 0.149 & 10.7 \\
\hline \multirow{4}{*}{ Ml101 } & \multirow{4}{*}{$9 / 24 / 2013$} & & & & MEP & 12.3 & 1.90 & 0.246 & Lost Sample \\
\hline & & 10.18 AM & 42.1239 & -87.0924 & TRM & 26.0 & 1.78 & 0.161 & Lost Sample \\
\hline & & 10:18 AIVI & 42.1239 & -87.0924 & MHY & 54.0 & 1.64 & 0.111 & 36.9 \\
\hline & & & & & B- & 74.6 & 1.61 & 0.134 & 37.3 \\
\hline M110? & $9 / 24 / 2013$ & $2.45 \mathrm{PM}$ & 418847 & 874898 & SRF & 1.8 & 1.84 & 0.190 & 13.9 \\
\hline IVIIIOL & $5 / \angle 4 / \angle 013$ & $2.45 \mathrm{PIVI}$ & 41.0041 & -01.4090 & B- & 12.0 & 1.81 & 0.198 & 21.3 \\
\hline M1103 & $9 / 24 / 2013$ & 4.48 PM & 416866 & -87.3128 & SRF & 1.9 & 1.81 & 0.201 & 18.1 \\
\hline intios & $5 / \angle 4 / \angle 013$ & $4.40 \mathrm{PIVI}$ & 41.0000 & -01.2120 & B- & 12.7 & 1.80 & 0.194 & 15.0 \\
\hline & & & & & SRF & 1.8 & 1.79 & 0.194 & 17.7 \\
\hline MI104 & $9 / 24 / 2013$ & 6:36 PM & 41.6896 & $-8 / .01 / 0$ & B- & 10.5 & 1.83 & 0.208 & 11.0 \\
\hline M1105 & $9 / 24 / 2013$ & 8.56 PM & 421183 & 865159 & SRF & 1.9 & 1.87 & 0.244 & 18.6 \\
\hline IVIIUS & $9 / 24 / 2013$ & ৪:క6 PIVI & 42.1183 & -86.5159 & B- & 11.7 & 1.82 & 0.225 & 24.8 \\
\hline M1106 & $9 / 25 / 2013$ & $12 \cdot 29 \mathrm{AM}$ & 426793 & $-86,2405$ & SRF & 2.0 & 1.83 & 0.201 & 21.0 \\
\hline IVIIIOS & $9 / \angle 3 / \angle 013$ & $12.29 \mathrm{AlVI}$ & $42.6 / 93$ & -80.2405 & B- & 12.1 & 1.80 & 0.254 & 21.2 \\
\hline & & & & & SRF & 2.0 & 1.85 & 0.253 & 23.4 \\
\hline MI0/ & $9 / 25 / 2013$ & 2:52 AM & 43.0626 & $-86.2 / / 5$ & B- & 11.8 & 1.81 & 0.188 & 21.2 \\
\hline & & & & & MEP & 10.0 & 1.86 & 0.305 & 66.8 \\
\hline Mi108 & & $A \cdot 25 A M$ & 430934 & 865328 & TRM & 28.0 & 1.62 & 0.156 & 43.1 \\
\hline IVIIIOS & $9 / 25 / 2013$ & 4.25 AIVI & 43.0934 & $-80.53<8$ & MHY & 60.1 & 1.56 & 0.144 & 71.7 \\
\hline & & & & & B- & 94.4 & 1.61 & 0.147 & 31.6 \\
\hline M1109 & $0 / 25 / 2013$ & $6.50 \triangle A M$ & 13.2218 & 863649 & SRF & 1.9 & 1.98 & 0.212 & 22.5 \\
\hline IVIIOY & 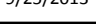 & 0.J0 Aivi & 43.2210 & -80.3649 & B- & 13.1 & 1.88 & 0.253 & 24.3 \\
\hline & & & & & SRF & 1.8 & 1.81 & 0.225 & 18.8 \\
\hline MI110 & $9 / 25 / 2013$ & 9.36 AM & 43.6371 & -866222 & no TRM & 17.2 & 1.68 & 0.152 & 29.1 \\
\hline IVIIIIO & $9 / 25 / 2013$ & 9.30 AIVI & $43.03 / 1$ & $-80.0 \angle 2 L$ & no TRM & 35.2 & 1.68 & 0.129 & 46.8 \\
\hline & & & & & B- & 66.4 & 1.62 & 0.133 & 42.6 \\
\hline MI111 & $9 / 25 / 2013$ & $12.32 \mathrm{PM}$ & 43.9565 & $-86,4943$ & SRF & 2.0 & 1.82 & 0.237 & 16.1 \\
\hline IVIIII & $9 / 25 / \angle 013$ & IL:3Z PIVI & 43.9565 & -86.4943 & B- & 11.4 & 1.72 & 0.189 & 26.3 \\
\hline & & & & & MEP & 15.1 & 1.90 & 0.204 & 29.7 \\
\hline & & & & & TRM & 32.1 & 1.81 & 0.170 & 38.7 \\
\hline Ml112 & $9 / 25 / 2013$ & 7:12 PM & 44.7503 & -86.5798 & $\mathrm{DCL}$ & 44.2 & 1.66 & 0.124 & 16.7 \\
\hline & & & & & MHY & 139.9 & 1.57 & 0.112 & 49.0 \\
\hline & & & & & B- & 236.9 & 1.55 & 0.137 & 28.5 \\
\hline & & & & & MEP & 9.0 & 1.93 & 0.254 & 21.0 \\
\hline M1113 & $9 / 26 / 2013$ & $12.25 \mathrm{AM}$ & 44.9690 & -860370 & TRM & 18.2 & 1.83 & 0.153 & 29.0 \\
\hline IVIIIS & $9 / 2 b / 2013$ & 12:25 AIVI & 44.9690 & $-86.03 / 0$ & MHY & 58.1 & 1.63 & 0.120 & 46.8 \\
\hline & & & & & B- & 90.9 & 1.65 & 0.143 & 35.1 \\
\hline & & & & & MEP & 6.0 & 2.75 & 0.193 & 30.3 \\
\hline Ml114 & 9/26/2013 & 6:47 AM & 45.6099 & -87.0453 & TRM & 16.1 & 2.08 & 0.169 & 34.4 \\
\hline & & & & & B- & 26.4 & 2.00 & 0.157 & 28.4 \\
\hline Ml115 & $9 / 26 / 2013$ & 1:10 PM & 45.0945 & -87.5464 & SRF & 1.6 & 3.30 & 0.198 & 32.8 \\
\hline & & & & & B- & 14.9 & 3.02 & 0.185 & 31.1 \\
\hline M1116 & $9 / 26 / 2013$ & $3.24 \mathrm{PM}$ & 448078 & -877264 & SRF & 1.8 & 3.39 & 0.181 & 32.5 \\
\hline IVIIII6 & $y / \angle 6 / \angle 013$ & 3:24 PIVI & $44.80 / 8$ & $-81.1 / 264$ & B- & 11.3 & 3.43 & 0.191 & 34.7 \\
\hline & & & & & MEP & 10.1 & 1.79 & 0.179 & 24.0 \\
\hline MI117 & 9/27/2013 & 5:00 AM & 44.0645 & -87.4735 & TRM & 26.0 & 1.59 & 0.123 & 42.5 \\
\hline & & & & & B- & 58.2 & 1.58 & 0.122 & 42.8 \\
\hline Ml118 & $9 / 26 / 2013$ & 10:07 PM & 44.1368 & -87.5174 & SRF & 2.0 & 1.83 & 0.211 & 17.2 \\
\hline IVIII8 & $9 / \angle 6 / \angle 013$ & 10:0/ PIVI & & & B- & 13.5 & 1.71 & 0.161 & 15.6 \\
\hline M1119 & $9 / 26 / 2013$ & $11.20 \mathrm{PM}$ & 44.0853 & -87.5888 & SRF & 2.0 & 1.82 & 0.198 & 20.5 \\
\hline IVIIIIT & $9 / \angle 0 / \angle 013$ & $11.20 \mathrm{PIVI}$ & 44.0833 & -81.3880 & B- & 11.7 & 1.82 & 0.171 & 18.6 \\
\hline M120 & $9 / 27 / 2013$ & 3.39. AM & 437481 & -876704 & SRF & 5.0 & 1.87 & 0.173 & Lost Sample \\
\hline IVIIILO & $9 / 21 / 2013$ & 3.39 AIVI & $43 . / 481$ & -81.0704 & B- & 12.5 & 1.83 & 0.203 & 7.7 \\
\hline & & & & & MEP & 13.5 & 1.79 & 0.265 & 54.9 \\
\hline N118 & $9 / 24 / 2013$ & $5.23 \triangle M$ & 7331 & 869981 & TRM & 37.3 & 1.62 & 0.133 & 35.9 \\
\hline TVIII & $9 /<4 / 2013$ & 5:Z3 AIVI & 42.1331 & -80.9981 & MHY & 100.3 & 1.58 & 0.131 & 55.5 \\
\hline & & & & & B- & 155.0 & 1.54 & 0.126 & 31.1 \\
\hline & & & & & MEP & 10.0 & 1.87 & 0.216 & 19.0 \\
\hline M134 & $9 / 25 / 2013$ & 2.07 PM & 440899 & -867667 & TRM & 21.1 & 1.74 & 0.150 & 41.5 \\
\hline 101134 & J & $2.07 \mathrm{r} / \mathrm{vi}$ & 44.0057 & -00.1001 & MHY & 90.0 & 1.63 & 0.132 & 36.1 \\
\hline & & & & & B- & 155.0 & 1.62 & 0.129 & 35.7 \\
\hline & & & & & MEP & 8.3 & 1.91 & 0.221 & 23.6 \\
\hline M147 & $9 / 26 / 2013$ & $2.08 \mathrm{AM}$ & 45.1782 & $-86,3748$ & TRM & 24.1 & 1.79 & 0.154 & 27.2 \\
\hline TVIII/ & $9 / \angle 0 / \angle 013$ & 2:U8 AIVI & $43.1 / 82$ & $-80.3 / 48$ & MHY & 112.3 & 1.61 & 0.120 & 37.5 \\
\hline & & & & & B- & 187.2 & 1.56 & 0.137 & 26.2 \\
\hline & & & & & MEP & 8.0 & 2.21 & 0.193 & 26.8 \\
\hline Ml49 & 9/26/2013 & 7:58 AM & 45.4913 & -87.0330 & TRM & 26.0 & 1.83 & 0.142 & 38.8 \\
\hline & & & & & B- & 41.1 & 1.83 & 0.149 & Lost Sample \\
\hline & & & & & MEP & 8.1 & 3.19 & 0.164 & 28.1 \\
\hline Ml50 & $9 / 26 / 2013$ & 11:38 AM & 45.1163 & -87.4158 & TRM & 22.1 & 2.09 & 0.143 & 16.9 \\
\hline & & & & & B- & 29.6 & 2.16 & 0.161 & 29.4 \\
\hline
\end{tabular}




\section{Citations:}

1) National Geophysical Data Center, 1996. Bathymetry of Lake Michigan. National Geophysical Data Center, NOAA. doi:10.7289/V5B85627 [11/25/2020]. 